OPEN ACCESS

Edited by:

Kai Deng,

Sun Yat-Sen University, China

Reviewed by:

Xiaohong Liu,

Sun Yat-Sen University, China

Xuehui Cai,

Harbin Veterinary Research Institute,

Chinese Academy of Agricultural

Sciences (CAAS), China

*Correspondence:

Jinhai Huang

jinhaih@tju.edu.cn

Lilin Zhang

lyliazhang@163.com

${ }^{\dagger}$ These authors have contributed equally to this work

Specialty section:

This article was submitted to Viral Immunology,

a section of the journal

Frontiers in Immunology

Received: 05 February 2021 Accepted: 23 April 2021

Published: 17 May 2021

Citation:

Song $Y$, Guo $Y$, Li X, Sun $R$,

Zhu M, Shi J, Tan Z, Zhang L

and Huang J (2021) RBM39

Alters Phosphorylation of C-Jun and Binds to Viral RNA to Promote

PRRSV Proliferation.

Front. Immunol. 12:664417. doi: 10.3389/fimmu.2021.664417

\section{RBM39 Alters Phosphorylation of c-Jun and Binds to Viral RNA to Promote PRRSV Proliferation}

\author{
Yinna Song ${ }^{1 \dagger}$, Yanyu Guo ${ }^{1 \dagger}$, Xiaoyang $\mathrm{Li}^{1}$, Ruiqi Sun ${ }^{1}$, Min Zhu ${ }^{1}$, Jingxuan Shi ${ }^{1}$, Zheng Tan ${ }^{1}$, \\ Lilin Zhang ${ }^{1,2^{*}}$ and Jinhai Huang ${ }^{1,2^{*}}$ \\ ${ }^{1}$ School of Life Sciences, Tianjin University, Tianjin, China, ${ }^{2}$ Tianjin Key Laboratory of Function and Application of Biological \\ Macromolecular Structures, Tianjin University, Tianjin, China
}

As transcriptional co-activator of $\mathrm{AP}-1 / \mathrm{Jun}$, estrogen receptors and $\mathrm{NF}-\mathrm{\kappa B}$, nuclear protein RBM39 also involves precursor mRNA (pre-mRNA) splicing. Porcine reproductive and respiratory syndrome virus (PRRSV) causes sow reproductive disorders and piglet respiratory diseases, which resulted in serious economic losses worldwide. In this study, the up-regulated expression of RBM39 and down-regulated of inflammatory cytokines (IFN- $\beta$, TNF $\alpha, N F-\kappa B, I L-1 \beta, I L-6)$ were determined in PRRSVinfected 3D4/21 cells, and accompanied with the PRRSV proliferation. The roles of RBM39 altering phosphorylation of C-Jun to inhibit the AP-1 pathway to promote PRRSV proliferation were further verified. In addition, the nucleocytoplasmic translocation of RBM39 and c-Jun from the nucleus to cytoplasm was enhanced in PRRSV-infected cells. The three RRM domain of RBM39 are crucial to support the proliferation of PRRSV. Several PRRSV RNA (nsp4, nsp5, nsp7, nsp10-12, M and N) binding with RBM39 were determined, which may also contribute to the PRRSV proliferation. Our results revealed a complex mechanism of RBM39 by altering c-Jun phosphorylation and nucleocytoplasmic translocation, and regulating binding of RBM39 with viral RNA to prompt PRRSV proliferation. The results provide new viewpoints to understand the immune escape mechanism of PRRSV infection.

Keywords: RBM39, c-Jun, porcine reproductive and respiratory syndrome virus, AP-1, phosphorylation, interferon

\section{INTRODUCTION}

RNA-binding proteins (RBPs) have been found in all living organisms (1). Through binding RNAs, RBPs assemble in ribonucleoprotein complexes, which dictate the fate and the function of virtually every cellular RNA molecules. RBPs can bind single-stranded or double-stranded RNAs, and play an important role in regulating RNA metabolism and gene expression as post-transcriptional regulators $(2,3)$. RBPs are involved in biological processes such as RNA transcription, editing, splicing, transportation and positioning, stability and translation (4). With the extensive reports on the post-transcriptional regulatory mechanism of RBPs, more and more scholars are focusing on their complicated functions. The target RNA of RBPs is variable and diverse, and they have ability to bind to different region of mRNA [such as exons, introns, untranslated regions (UTRs)], or interact with other types of RNA, including non-coding RNAs (5), microRNAs, small interference RNAs 
(siRNA), t-RNAs, small nucleolar RNA (snoR-NA), telomerase RNA, conjugant small nuclear RNA (snRNA) and the RNA part of signal recognition particles (SRP RNA or 7SL RNA (6-8). These non-coding RNAs form a wide range of secondary structures, which combine with RBP and regulate processes such as RNA splicing, RNA modification (9), protein localization, translation, and maintenance of chromosome stability $(10,11)$. The functional effects of conventional RBPs depend on the target RNA-RNP complex formation. Simultaneously, the RNP complex helps RNA processing, translation, export and localization $(10,12)$.

RBM39 (RNA binding motif protein 39), also called CAPER, HCC1.3/1.4,Caper $\alpha, F S A P 59, R N P C 2$ (13), is a nuclear protein that is involved in precursor mRNA (pre-mRNA) splicing (14). RBM39 was first identified as an auto-antigen in a hepatocellular carcinoma patient (15). In addition, RBM39 is a transcriptional co-activator of activating protein-1 (AP-1/Jun), estrogen receptors (eg, steroid nuclear receptors ESR1/ER-alpha and ESR2/ER- $\beta$ ) and NF- $\mathrm{KB}(14,16)$. Based on the previous research, the function of RBM39 has been associated with malignant progression in a number of cancers (15). Previous studies have reported RBM39 as a proto-oncogene with important roles in the development and progression of multiple types of malignancies (17). RBM39 is relevant to numerous precursor messenger RNA (pre-mRNA) splicing factors and provide regulating action of a quantity of signal pathway $(15,18)$. Degradation of RBM39 led to aberrant premRNA splicing, including intron retention and exon skipping in hundreds of gene (15). RBM39 has three RNA recognition motif (RRM) and the C-terminal of RRM3 domain belongs to the U2AF homology motif family (UHM), which mediate proteinprotein interactions through a short tryptophan-containing peptide known as the UHM-ligand motif (ULM) $(14,16)$. However, RBM39 impacting virus proliferation and its role in innate immunity are largely unknown.

The activation protein-1 (AP-1) transcription factors are immediate early response genes involved in a diverse set of transcriptional regulatory processes $(19,20)$. The AP-1 complex is mainly composed of a Fos family member and a Jun family member encoded by the proto-oncogene, which bind to the DNA target sequence in the form of a homologous or heterodimer complex to regulate the expression of the target gene $(21,22)$. The AP-1 complex consists of Fos family members, Jun family members, activating transcription factor (ATF) and musculoaponeurotic fibrosarcoma (MAF) (21). The Fos family contains c-Fos, v-Fos, Fos-B, Fra-1 and Fra-2 (21); the Jun family is composed of c-Jun, v-Jun, Jun-B, and Jun-D (19); ATF includes ATF2, ATF/LRF1, B-ATF, JDP1 and JDP2 (23); and MAF comprises C-Maf, MafB, MafA, MafG/F/K and Nrl (23). The c-Jun is a central component of all AP-1 complexes and members of the basic region-leucine zipper (bZIP) family of sequence-specific dimeric DNA-binding proteins $(19,24)$. In addition, the c-Jun is a transcription factor that recognizes the AP-1 complex and binds to the enhancer heptamer motif 5'TGA[CG]TCA-3' (termed AP-1 sites) found in a variety of promoters $(14,19)$. The c-Jun gene is expressed in multiple cell at low levels, and its expression is in elevated response to many stimuli, including growth factors, cytokines, foreign materials and viral infection (25). Certain stimuli of cells result in activation of the JNK (c-Jun N-terminal kinase) and p38 groups of MAPKs, followed by the c-Jun were phosphorylated by JNKs at two positive regulatory sites (serine 63 and 73) residing within its amino-terminal activation domain $(9,19)$.

Porcine reproductive and respiratory syndrome virus (PRRSV) is a critical pathogen in pig and can trigger a serious negative impact on the economic development of pigs $(26,27)$, which mainly causes sow reproductive disorders and piglets respiratory diseases, thus resulting in serious economic losses in the world (28). It is a variety of capsule single-stranded positive-chain virus of arteritis virus (29). Infections by PRRSV often result in increased expression of multiple genes and delayed low-level induction of antiviral cytokines, thereby destroying the early endogenous immune response $(30,31)$. The 15kb PRRSV genome is expressed through a set of subgenomic mRNA transcripts, each used for the translation of one or two open reading frames (ORFs) $(32,33)$. The full-length viral RNA encodes two large nonstructural polyproteins, ppla and pplab (34), which are processed by viral proteases to release 14 non-structural proteins (35), including four proteases (nsp1 $\alpha$, nsp1 1 , nsp2 and nsp4), the RNA-dependent RNA polymerase (nsp9), a helicase (nsp10) and an endonuclease (nsp11) $(33,36)$. In this study, the RBM39 participated in PRRSV RNA binding and viral proliferation was investigated. The dual regulation effects of RBM39 compete with c-Jun to down-regulated IFN- $\beta$ production and prompt PRRSV proliferation by stabilizing and binding with viral RNA were illustrated.

\section{MATERIALS AND METHODS}

\section{Cell and Virus Cultures}

Human embryonic kidney 293 T cells (HEK293 T) and HeLa cells were cultured in Dulbecco's modified essential medium (DMEM), porcine alveolar macrophages (PAM) cell and PAM cell line 3D4/21 in RPMI-1640 medium (Biological Industries) in incubator at $37^{\circ} \mathrm{C}$ with $5 \% \mathrm{CO}_{2}$. All media were obtained from Biological Industries and supplemented with $10 \%$ fetal bovine serum (FBS), $100 \mathrm{U} / \mathrm{ml}$ penicillin, and $100 \mu \mathrm{g} / \mathrm{ml}$ streptomycin. The PRRSV was preserved in our laboratory and produced by transfection of 3D4/21 cells with the PRRSV-JXwn06 infectious clone plasmid. In this study, the PRRSV was used with a titer of $10^{4} \mathrm{PFU} / \mathrm{ml}$. Sendai virus $(\mathrm{SeV})$ and vesicular stomatitis virus (VSV) was preserved in our laboratory.

\section{Antibodies and Reagents}

Murine RBM39 pAb was prepared by immunizing mice with the purified protein. Anti-PRRSV nsp2 antibody was a gift from Prof. Jun Han of China Agricultural University and Rabbit antiPRRSV-N protein $\mathrm{pAb}$ was purchased from YBio Technology (YB-23941R). Mouse anti-Flag/ $\beta$-actin/GADPH $\mathrm{mAb}$ and Peroxidase-Conjugated Goat anti-Rabbit/Mouse IgG $(\mathrm{H}+\mathrm{L})$ were purchased from Yeasen Technology. Rabbit anti-phospho-JNK1 
$+2+3$ (Thr183+Tyr185) pAb was purchased from Bioss (bs1640R); Rabbit anti-c-Jun mAb was purchased from Abways Technology (CY5290); Rabbit anti-phospho-c-Jun (Ser73) mAb was purchased from Cell Signaling Technology (3270T); Rabbit anti-HA pAb, Goat anti-Mouse IgG $(\mathrm{H}+\mathrm{L})$ Alexa Fluor 555 and Goat anti-Mouse/Rabbit IgG $(\mathrm{H}+\mathrm{L})$ FITC was purchased from Invitrogen; Mouse anti-Flag/HA-tags beads was purchased from Abmart. Phosphatase inhibitors were purchased from APE $\times$ BIO. Quick CIP were purchased from Biolabs.

\section{Plasmids}

RBM39 and c-Jun gene was cloned from 3D4/21 cells cDNA as template. Flag/HA-RBM39 and Flag/HA-c-Jun plasmids were constructed by seamless cloning technology. Flag-RRM1, RRM2, RRM3, $\Delta R R M 1, \Delta R R M 2, \Delta R R M 3, \Delta R R M, \Delta$ NLS plasmids were constructed by reverse amplification by Flag-RBM39 plasmid as template. Plasmid pET-28a-RBM39-X7 that delete the complete 5 -terminal NLS sequence was also constructed by seamless cloning method, to purify the RBM39 protein and make its antibodies. AP-1/IFN- $\beta /$ Renilla luciferase reporter plasmids were constructed as previously described (29). The primers used above are shown in Table $\mathbf{1}$.

\section{Transient Transfection of Eukaryotic Expression Plasmid Into Cells}

Previous to transient transfection, adherent HEK293T, HeLa or 3D4/21 cells were seeded in a 6/12/24-well plate (Corning Inc, Corning, NY, USA). Cells were transfected at the appropriate confluence with eukaryotic expression plasmid. Briefly, $10 \mu \mathrm{M}$ polyethyleneimine (PEI) was preheated at $85^{\circ} \mathrm{C}$ and replace the old medium with Reduced-Serum Medium (Opti-MEM, Thermo Fisher Scientific) before transfection $1 \mathrm{~h}$ in advance. Add a suitable equal volume of medium to the two centrifuge tubes, then add the appropriate plasmid and PEI, shake and mix, and place at room temperature for 5 minutes. Then, mix and place them at room temperature for 20-30 minutes. Afterwards, add the mixture to the cells and incubate the cells in $37^{\circ} \mathrm{C}$ incubator containing 5\% $\mathrm{CO}_{2}$. After 4-6 hours, discard the mixed solution and replace to complete medium, and continue to grow cells for 24-48 h.

\section{Western Blot}

Cell samples were lysed by radioimmunoprecipitation assay (RIPA) (Solarbio) added with protease inhibitor cocktail phenylmethanesulfonyl fluoride (PMSF, Solarbio). Cells lysis supernatants were added to $5 \times$ loading buffer, following boiled for $10 \mathrm{~min}$ and separated by SDS-PAGE. The separated proteins were transferred onto a polyvinylidene fluoride (PVDF) blotting membrane (GE Healthcare) and blocked for $1 \mathrm{~h}$ with $5 \%$ skimmed milk in $1 \times$ TBST (Tris-buffered saline containing $0.05 \%$ Tween-20) at room temperature, followed by incubation with the primary antibodies diluted in $1 \times \mathrm{TBST}$ at $4^{\circ} \mathrm{C}$ overnight and secondary HRP-conjugated antibodies diluted in $1 \times$ TBST for $1 \mathrm{~h}$ at room temperature. Finally, the membrane was detected through Pierce ECL Western Blotting Substrate (Thermo Scientific, Waltham, MA, USA) and exposed through Chemi Doc XRS Imaging System (BIO-RAD, USA).

\section{Quantitative Real-Time PCR}

Quantification of the relative levels of gene expression was performed using qRT-PCR. Total RNAs were extracted by RNAiso plus (Takara). The cDNA was synthesized with EasyScript First-Strand cDNA Synthesis SuperMix according to the manufacturer's instructions (TransGene). The relative levels of gene expression were analyzed by QTOWER3G IVD Fluorescent Quantitative PCR System (Jena, Germany) using TransStart Top Green qPCR SuperMix (TransGene) with threestep amplification and the data were calculated by the comparative cycle threshold $\left(\mathrm{C}_{\mathrm{T}}\right)$ method $\left(2^{-\Delta \Delta \mathrm{Ct}}\right)$. The thermal cycler program consisted of $95^{\circ} \mathrm{C}$ for $10 \mathrm{~min}$ and then

TABLE 1 | Primers used in PCR amplification.

\begin{tabular}{|c|c|}
\hline Primer name & Primer sequence $\left(5^{\prime}-3^{\prime}\right)$ \\
\hline & R: TCATCGTCTACTTGGAACCAG \\
\hline & R: TCATCGTCTACTTGGAACCAG \\
\hline \multirow[t]{2}{*}{ P-C-Jun } & F: GGATCCATGACTGCAAAGATGGAAACGACCTTCTAC \\
\hline & R: GGATCCTCAAAACGTTTGCAACTGCTGCGTTAG \\
\hline pCMV-RBM39 & F: CCAGTCGACTCTAGAGGATCCATGGCAGACGATATTGATATTGAAGC \\
\hline & R: CAGGGATGCCACCCGGGATCCTCAAAACGTTTGCAACTGCTGC \\
\hline \multirow[t]{2}{*}{ pET-28a-RBM39-X7 } & F: CAGCAAATGGGTCGCGGATCCATGGCAGACGATATTGATATTGAAGC \\
\hline & R: GTGGTGGTGGTGGTGCTCGAGTCGTCTACTTGGAACCAGTAGTT \\
\hline \multirow[t]{2}{*}{ RRM1 off } & F: AAGAGATGCAGAAAAAAACAGAGCTGCAGC \\
\hline & R: TGCATCTCTTTCCTCAGGAGTTAGATTATC \\
\hline RRM2off & F: TGCTGGACCTCGTACTGATGCTTCCAGTGC \\
\hline & R: CATTGCTTCAATATCAATATCGTCTGCCAT \\
\hline
\end{tabular}


45 cycles at $95^{\circ} \mathrm{C}$ for $15 \mathrm{~s}, 58^{\circ} \mathrm{C}$ for $30 \mathrm{~s}$, and $72^{\circ} \mathrm{C}$ for $30 \mathrm{~s}$. The $\beta$ actin was used as an internal control for normalization. All the primers used for quantitative real-time PCR are shown in Table 2.

\section{Dual Luciferase Reporter Assay}

HEK293 T cells plated to 24 -well plates at 70\%-80\% confluence were transfected with RBM39, AP-1 luciferase reporter plasmid, PRRSV-JXwn06 infectious clone plasmid, siRBM39 or sic-Jun (Sigma) and the Renilla luciferase control reporter plasmid was used as a reference. The cells were collected and lysed at $24 \mathrm{~h}$ post-transfection, and the cell supernatant was used to detect luciferase activity through Dual Luciferase Reporter Gene Assay Kit (Yeasen Technology) according to the manufacturer's instructions.

\section{Immunoprecipitation (IP) and Co- Immunoprecipitation (co-IP) Assays}

HEK293 T Cells cultivated in six-well plates were transfected or co-transfected with appropriate eukaryotic expression plasmid. The cells were harvested and resuspended by PBS $24 \mathrm{~h}$ posttransfection, and lysed on ice for $15 \mathrm{~min}$ in $400 \mu \mathrm{l}$ RIPA lysis buffer. Then the samples were centrifuged at $12,000 \mathrm{rpm}$ for $5 \mathrm{~min}$. We took out the $50 \mu \mathrm{L}$ supernatant in new tube, added $10 \mu \mathrm{L}$ loading buffer and boiled them to make input samples. Subsequently, anti-Flag-labeled beads (Sigma) were activated by RIPA and added the rest of the cell lysis supernatant. The reaction mixture was incubated at $4^{\circ} \mathrm{C}$ overnight. The beads were washed three times with lysis buffer containing PMSF for 5 min each time and added $50 \mu \mathrm{L}$ lysates and $10 \mu \mathrm{L}$ loading buffer, boiled for $10 \mathrm{~min}$ to make IP samples. Finally, the proteins bound to the beads were separated via SDS-PAGE, transferred to PVDF membrane, and detected with the proper antibodies.

TABLE 2 | Primers used in quantitative real-time PCR.

\begin{tabular}{|c|c|}
\hline Primer name & Primer sequence (5'-3') \\
\hline \multirow[t]{2}{*}{ Q-RBM39 } & F: CCAAATGCCAAAGAACC \\
\hline & R: GAATGTGCCAAGAAAGC \\
\hline \multirow[t]{2}{*}{ Q-nsp2 } & F: CAGCCTTATGACCCCAACCAG \\
\hline & R: TGGGCAAAGTCCCCTGTACCAA \\
\hline \multirow[t]{2}{*}{ Q-N } & F: CAGTCAATCAGCTGTGCCAAA \\
\hline & R: ATCTGACAGGGCACAAGTTCCA \\
\hline \multirow[t]{2}{*}{ Q-C-Jun } & F: ACGACCTTCTACGACGATG \\
\hline & R: TGGTGATGTGCCCGTTA \\
\hline \multirow[t]{2}{*}{ Q- $\beta$-actin } & F: CAAATGCTTCTAGGCGGACT \\
\hline & R: TGCTGTCACCTTCACCGTTC \\
\hline \multirow[t]{2}{*}{ Q-IFN- $\beta$} & F: GCAGTATTGATTATCCACGAGA \\
\hline & R: TCTGCCCATCAAGTTCCAC \\
\hline \multirow[t]{2}{*}{$Q-N F-\kappa B$} & F: GTGTGTAAAGAAGCGGGACCT \\
\hline & R: CACTGTCACCTGGAAGCAGAG \\
\hline \multirow[t]{2}{*}{ Q-TNF- $\alpha$} & F: GAGATCAACCTGCCCGACT \\
\hline & R: CTITCTAAACCAGAAGGACGTG \\
\hline \multirow[t]{2}{*}{ Q-IL-6 } & F: ACTGGCAGAAAACAACCTGA \\
\hline & R: CCTCGACATTTCCCTTATTGCT \\
\hline \multirow[t]{2}{*}{ Q-IL-1 $\beta$} & F: TGTTCTGCATGAGCTाTGTG \\
\hline & R: TCTGGGTATGGCTITCCTTAG \\
\hline
\end{tabular}

\section{Indirect Immunofluorescent Assay (IFA) and Confocal Microscopy}

Adherent HEK293T, HeLa or 3D4/21 cells grown on glass coverslips in 12 -well plates at $30 \%$ to $50 \%$ confluence were transfected with plasmids expressing RBM39 and/or c-Jun. The empty vector plasmid was used as a negative control (NC). Subsequently, 3D4/21 cells were infected with PRRSV at a multiplicity of infection (MOI) of 0.4 . At $24 \mathrm{~h}$ post-transfection or post-infection, the cells washed with $1 \times$ phosphate-buffered saline (PBS), fixed with $4 \%$ paraformaldehyde for $30 \mathrm{~min}$ at room temperature, permeabilized with $0.5 \%$ Triton X-100 for $15 \mathrm{~min}$ and blocked with $5 \%$ bovine serum albumin (BSA) dissolved in $1 \times \mathrm{PBS}$ containing $0.05 \%$ of Tween-20 (PBST) for $2 \mathrm{~h}$ at room temperature. Anti-HA (hemagglutinin), anti-Flag and antiPRRSV-N primary antibodies were diluted in 2\% BSA and incubated with slides overnight at $4^{\circ} \mathrm{C}$. Following a wash performed with PBS 3 times for 5 min each time, the cells were incubated with fluorescein isothiocyanate (FITC)-conjugated goat anti-mouse/rabbit IgG or IF555-conjugated goat anti-mouse IgG secondary antibody diluted in $2 \%$ BSA at $37^{\circ} \mathrm{C}$ for $1 \mathrm{~h}$ in a humidified chamber. The slides were washed with PBST and nuclei were counterstained with Hoechst 33258 dye (Solarbio) for $5 \mathrm{~min}$. Confocal images were collected with confocal laser scanning microscope (UltraView Vox, PerkingElmer) and taken at $40 \times$ or $100 \times$ magnification (Olympus).

\section{RNA Interference}

A small interfering RNA (siRNA) assay, which was synthesized from Sigma (Table 3), was performed through siRNA targeting the knockdown of RBM39 (siRBM39) or c-Jun (sic-Jun) gene, and negative control (NC) was used as control groups. For siRNA transfection, 3D4/21 cells plated in 12-well plates and grown to $70 \%-80 \%$ confluence were transfected with $50 \mathrm{nmol}$ of siRNAs using $10 \mu \mathrm{M}$ PEI. The cells were infected with PRRSV at 0.4 MOI $24 \mathrm{~h}$ post-transfection and incubated for additional 24 h. Subsequently, cells were collected for qRT-PCR and Western blotting analysis to detect the expression levels.

\section{RNA Immunoprecipitation (RIP)}

$3 \mathrm{D} 4 / 21$ cells plated in 6-well plates and grown to $70 \%-80 \%$ confluence were transfected with $2 \mu \mathrm{g}$ HA-RBM39 or FlagRBM39 plasmids. Then the cells were infected with $0.4 \mathrm{MOI}$

TABLE 3 | Primers used in RNA interference assay.

\begin{tabular}{ll}
\hline Primer name & \multicolumn{1}{c}{ Primer sequence (5'-3') } \\
\hline Negative control (RBM39) & F: UUCUCCGAACGUGUCACGUTT \\
siRNA-RBM39-1308 & R: ACGUGACACGUUCGGAGAATT \\
FiRNA-RBM39-1631 & R: GGAUUUGAGCUCGCAGGAATT \\
Negative control (C-Jun) & F: AAACAUGUUAGAGAGUUGGTT \\
SiRNA-C-Jun-197 & F: UUCUCCGCUCUCUAACAUGUUUTT \\
SiRNA-C-Jun-261 & R: ACGUGACACGUUCGGAGAATT \\
& F: AGGUCGUUUCCAUCUUUGCTT \\
& R: GCAAAGAUGGAAACGACCUTT \\
& F: UUACUGUAGCCGUAGGCACTT \\
R: GUGCCUACGGCUACAGUAATT
\end{tabular}


PRRSV $12 \mathrm{~h}$ after transfection. After another 24 hours, the cells were collected and lysed with $400 \mu \mathrm{l}$ RIPA supplemented with murine RNase inhibitor (1:100). Part of the supernatant was used for immunoblotting analysis, and the remaining supernatant was incubated with $\mathrm{HA} /$ Flag-tags beads overnight at $4^{\circ} \mathrm{C}$. The coprecipitated RNAs were extracted, reverse transcribed and detected through PCR via corresponding primers.

\section{Statistical Analysis}

Data were presented as the mean of three independent replicates. Statistical significance was analyzed by the unpaired t-test using Origin and GraphPad Prism software. Statistical differences were considered to be statistically significant at $\mathrm{P}$-value $<0.05\left({ }^{*} \mathrm{p}<\right.$ $\left.0.05 ;{ }^{* *} \mathrm{p}<0.01 ;{ }^{* *} \mathrm{p}<0.001 ;{ }^{* * *} \mathrm{p}<0.0001\right)$.

\section{RESULTS}

\section{Upregulation of RBM39 in 3D4/21 Cells by PRRSV Infection}

PRRSV infection can result to upregulation or downregulation of many genes in cells. To find out genes that displayed significant changes in transcription after PRRSV infection, a transcriptome sequencing analysis derived from PAM cell was performed. RBM39 was found to be significantly upregulated after PRRSV infection by statistical analysis (Figure 1A). Through the analysis of RBM39 gene domain, it is known that the protein is conserved in mammals (Figure 1B). To investigate the levels of RBM39 accumulation during viral infection, we infected 3D4/21 and PAM cells with PRRSV at 0.4 MOI. After collecting cells and extracting total RNA at different time points ( $12 \mathrm{~h}, 24 \mathrm{~h}$ and $36 \mathrm{~h}$ ) post-infection, mRNA abundance of RBM39 was analyzed by quantitative reverse transcription-PCR (qRT-PCR). Compared with the control group, the mRNA levels of RBM39 significantly increased at three different time points after PRRSV infection (Figure 1C). Furthermore, to analyze the effect of PRRSV infection on the protein expression level of RBM39 gene, we collected cells infected with PRRSV for different time periods ( $0 \mathrm{~h}, 12 \mathrm{~h}, 24 \mathrm{~h}$ and $36 \mathrm{~h} ; 0.4 \mathrm{MOI})$ or at different multiplicity of infection $(0,0.1,0.2$ and $0.4 ; 24 \mathrm{~h})$, followed by Western blot analysis with antibody to RBM39. The results showed that the protein expression in the cellular level of RBM39 after PRRSV infection was a dramatic increase compared with control groups (Figures 1D and S2A) and increased over time (Figures 1E and S2B). In addition, the results displayed that the protein

A
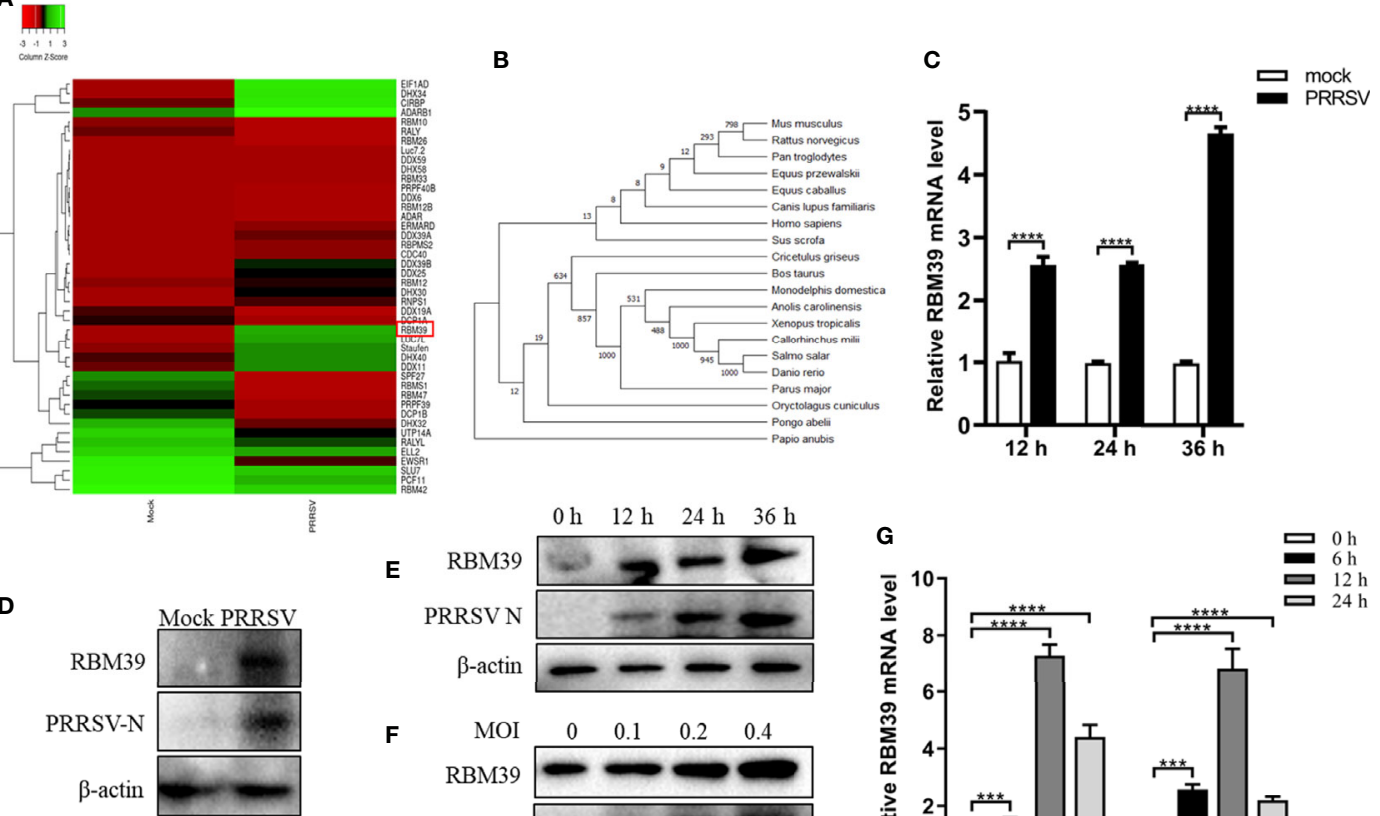

E

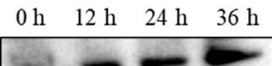

RBM39

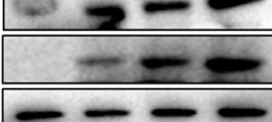

G

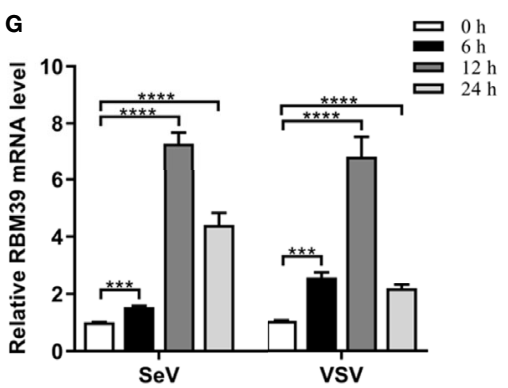

FIGURE 1 | Upregulation of RBM39 in 3D4/21 cells by PRRSV infection. (A) Heat map of RNA binding protein differentially expressed genes made using online websites (http://www.heatmapper.ca/). (B) RBM39 evolutionary tree among different species. (C-E) 3D4/21 cells were subjected to mock infection or infected with 0.4 multiplicity of infection (MOI) PRRSV. (C) Cells were collected at the three time points (12, 24, $36 \mathrm{~h}$ ) and subjected to real-time RT-PCR to analyze the expression level of RBM39. (D) Cell lysates were collected at $24 \mathrm{~h}$ post-infection and subjected to Western blot analysis with antibody to RBM39 to analyze the protein expression. (E) The cells were collected at $0 \mathrm{~h}, 12 \mathrm{~h}, 24 \mathrm{~h}$ and $36 \mathrm{~h}$ post-infection; (F) The cells were infected with $0,0.1,0.2$ and $0.4 \mathrm{MOI}$ PRRSV respectively and were collected at $24 \mathrm{~h}$ post-infection, the RBM39 protein level was analyzed by WB. (G) 3D4/21 cells were infected with SeV and VSV. The cells were collected at 0, 6, 12, and 24 h post-infection, and the RBM39 mRNA level was analyzed by qRT-PCR. Data are representative of results from three independent experiments. The statistically significant at P-value was demonstrated in Statistical Analysis. 
expression level of RBM39 increased in a dose-dependent manner on MOI (Figures $\mathbf{1 F}$ and S2C). To verify the expression level of RBM39 after infection with other RNA viruses, we infected cells with $\mathrm{SeV}$ and VSV, and then extracted the total RNA of the cells at $0,6,12$ and $24 \mathrm{~h}$ after infection, and analyzed the mRNA level of RBM39 by qRT-PCR. The results showed that RBM39 mRNA levels increased significantly at four time points (Figure 1G).

\section{RBM39 Contributes to PRRSV Proliferation}

The effect of RBM39 on the virus has not been reported before. To explore the effect of RBM39 on PRRSV proliferation, the 3D4/21 and PAM cells were transfected with Flag-RBM39 expression plasmids or empty vector, followed by infected with 0.4 MOI PRRSV $12 \mathrm{~h}$ post-transfection. Total RNA was extracted from the cells at $24 \mathrm{~h}$ post-transfection, and subsequently, transcription levels of PRRSV nsp2 and $\mathrm{N}$ gene were analyzed by qRT-PCR. Compared with the control groups, the overexpression of RBM39 promoted the mRNA level of the PRRSV nsp2 and $\mathrm{N}$ gene (Figures 2A and S2D). Meanwhile, the virus titer in cells after transfection of the RBM39 plasmid was higher than the titer seen with the control cells (Figures 2B and S2E). Confocal microscopy showed that the RBM39 overexpression group can observe more green fluorescent signals in the field of view than the empty vector group (Figure 2C). To further figure out the enhancement of RBM39 on PRRSV, we collected cells at five different time point $(6 \mathrm{~h}$, 12 h, 24 h, 36 h, 48 h) after PRRSV infection respectively, and subjected to Western blot analysis with antibody to $\mathrm{N}$ and nsp2. The results of Western blot analysis revealed that the protein expression level of $\mathrm{N}$ and nsp2 increased with time (Figure 2D). Subsequently, to further verify the effect of RBM39 on viral proliferation, we designed RBM39 small interfering RNA (siRNA) that targeted RBM39 gene and inhibited the transcription and protein expression of RBM39. Consistent with previous results, knockdown of RBM39 reduced the PRRSV proliferation to some extent at the virus titer, transcription and protein levels (Figures 2E-I). The results indicate that changes in the transcription and translation levels of RBM39 can affect PRRSV proliferation and that RBM39 contributes to PRRSV proliferation.

In order to determine the effect of the three RRM domains of RBM39 on the proliferation of PRRSV, we constructed eight truncated forms of RBM39 via the reverse amplification method, namely Flag-RRM1, RRM2, RRM3, $\triangle \mathrm{RRM1}, \Delta \mathrm{RRM} 2, \Delta \mathrm{RRM} 3$, $\triangle \mathrm{RRM}, \triangle \mathrm{NLS}$ (Figure 3A). It is worth noting that the DNA sequence that connects each domain is preserved. 3D4/21 cells were transfected with these eight truncations and infected with

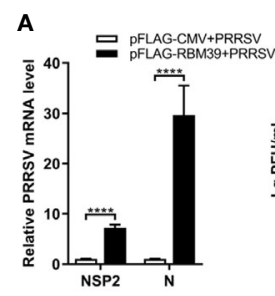

\section{(1)}

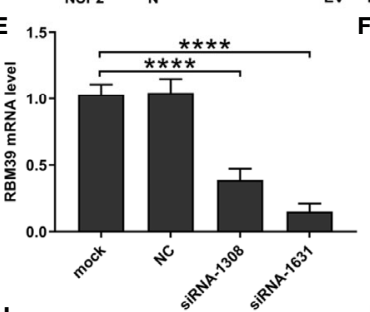

F

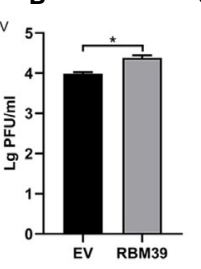

C
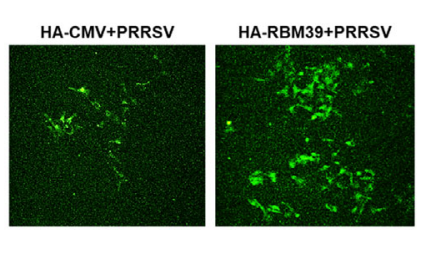

RBM39

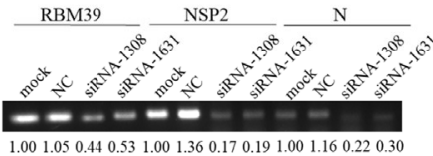

G

D

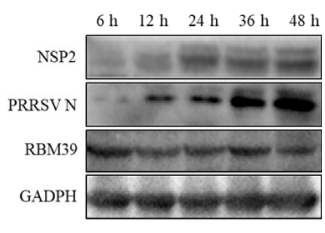

G

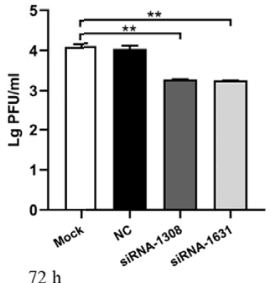

48
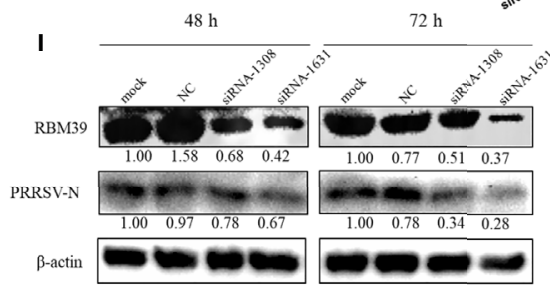

FIGURE 2 | RBM39 contributes to PRRSV proliferation. (A-D) 3D4/21 cells were transfected with Flag/HA-RBM39 or empty vector and infected with PRRSV at $12 \mathrm{~h}$ post-transfection. The infected cells were collected at the indicated times post-infection. (A) The mRNA loads of PRRSV N and nsp2 were tested by qRT-PCR. Detection of viral titers of PRRSV in cell supernatants of RBM39 overexpressed (B) or interfered (G) samples. (C) The cell slide was incubated overnight at $4^{\circ} \mathrm{C}$ with the antibody of PRRSV N and then incubated with FITC -labeled secondary antibody conjugate. The expression of PRRSV N was detected by Laser confocal fluorescence microscope. (D) PRRSV nsp2 and N protein expression were tested by WB. (E-I) The results showed 3D4/21 cells transfected with siRNA of RBM39, negative control (NC) or untransfected mock cells. The cells were infected with PRRSV at $12 \mathrm{~h}$ post-transfection and collected at $24 \mathrm{~h}$ post-infection. The mRNA levels of RBM39 (E, H), PRRSV N and nsp2 (H) were detected by qRT-PCR. (F) The agarose gel electrophoresis analysis after qRT-PCR. (I) RBM39 and PRRSV protein expression levels were examined by Western blot after 48 or $72 \mathrm{~h}$. Data are representative of results from three independent experiments. The statistically significant at P-value was demonstrated in Statistical Analysis. 

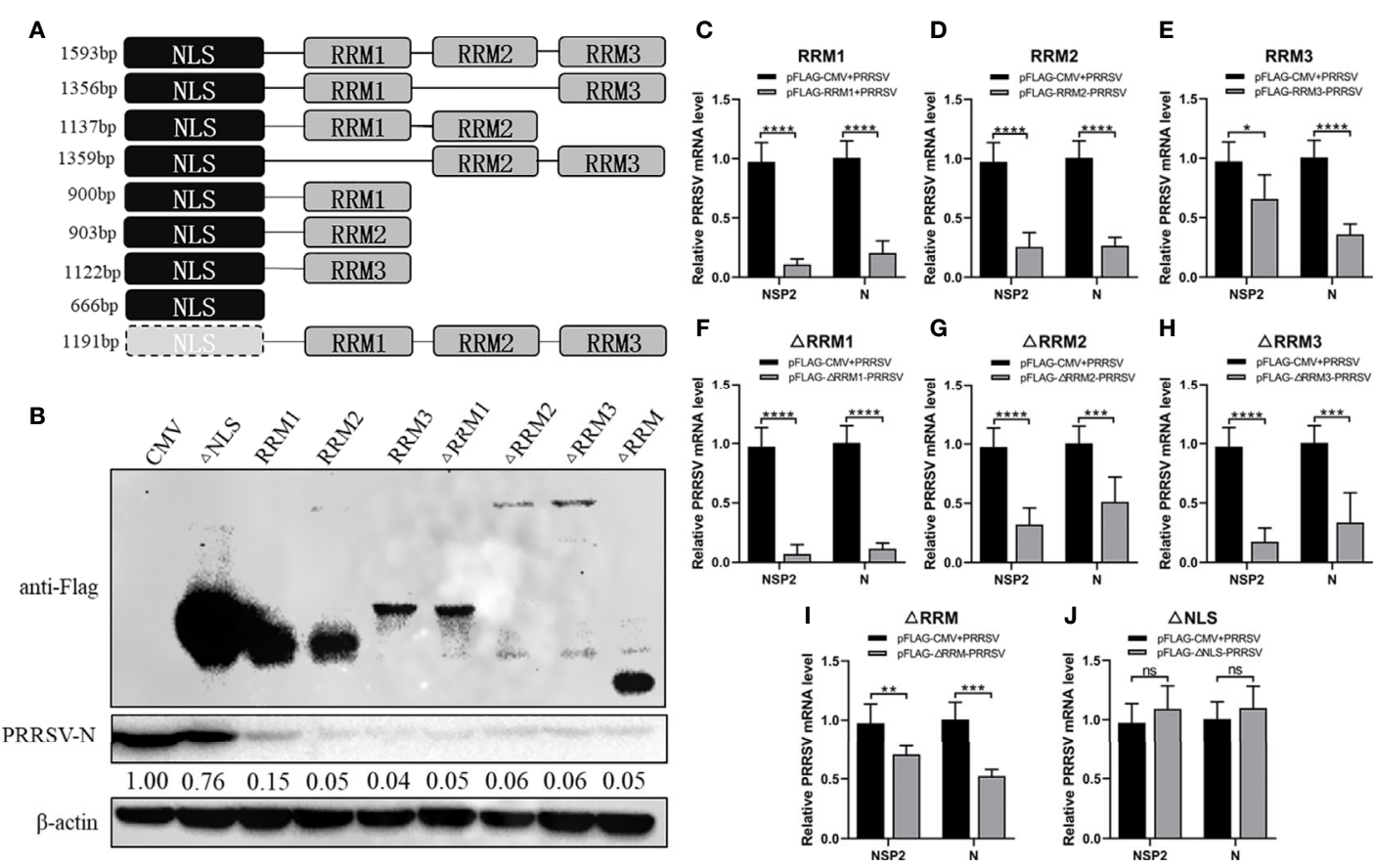

FIGURE 3 | The RRM domain of RBM39 is important to promote the proliferation of PRRSV. (A) The constructed 8 truncated forms of RBM39 via the reverse amplification method, namely Flag-RRM1, RRM2, RRM3, $\Delta R R M 1, \Delta R R M 2, \Delta R R M 3, \Delta R R M, \Delta N L S$. (B-J) 3D4/21 cells were transfected with 8 truncations and infected with PRRSV after 12 h. After 24 hours, the proliferation of PRRSV was detected by Western Blot analysis (B) and qRT-PCR (C-J). Data are representative of results from three independent experiments. The statistically significant at P-value was demonstrated in Statistical Analysis. ns, no significant difference.

the virus. After 24 hours, the proliferation of PRRSV was detected by qPCR and Western blot analysis. The results of qPCR and Western Blot analysis showed that compared with the control group, the proliferation of PRRSV in the experimental group transfected with Flag-RRM1, RRM2, RRM3, $\Delta$ RRM1, $\triangle R R M 2, \Delta R R M 3, \triangle R R M$ plasmid was inhibited (Figures 3BJ). However, the proliferation of PRRSV in the group transfected with Flag- $\Delta$ NLS had no significant difference (Figures 3B, J). It shows that the three RRM domains of RBM39 play an important role in promoting the proliferation of PRRSV.

\section{The Promotion of PRRSV Proliferation by RBM39 Is Related to Innate Immunity}

Previous studies showed that RBM39 is beneficial to PRRSV proliferation. To test the possibility of RBM39 negative regulatory effect on innate immunity, we examined the mRNA levels and concentration of some transcription factors and cytokines related to innate immunity, such as NF- $\kappa B$, TNF- $\alpha$, IFN- $\beta$, IL- $1 \beta$, IL- 6 . Cytokine concentration is detected by ELISA kit (Porcine TNF- $\alpha /$ IFN- $\beta /$ IL-1 $\beta /$ IL-6 ELISA KIT, Solarbio). The results showed that overexpression of RBM39 drastically decreased the transcription and secretion levels of these genes in 3D4/21 cells (Figures 4A, B). To investigate whether RBM39 impacts IFN- $\beta$-related signaling pathway, HEK293T cells were transfected with HA-RBM39 and PRRSV infectious clone plasmid or co-transfected with them. Subsequently, we measured the activity of the IFN- $\beta$ promoter reporter gene and found that the activation was increased by PRRSV stimuli but reduced by RBM39 overexpression compared with the control group (Figure 4C). Most important of all, when RBM39 and PRRSV co-transfected, the activity is lower than that of the group transfected with PRRSV alone, indicating that the overexpression of RBM39 may promote PRRSV proliferation by inhibiting the IFN- $\beta$ signaling pathway (Figure 4C). In addition, the mRNA and secretion levels of these genes related to innate immunity were upregulated when expression of RBM39 was disturbed by the introduction of siRNA (Figures 4D-F). Ulteriorly, we found out that RBM39 was capable of attenuating the inhibitory effect of interferon on PRRSV (Figure 4G) and increasing the virus titer at the same time (Figure $\mathbf{4 H}$ ). In conclusion, these data demonstrated that RBM39 might negatively regulate antiviral immune responses and resulting in the promotion of PRRSV proliferation.

\section{Interaction and Co-Localization Between RBM39 and C-Jun}

Previous research and mass spectrometry (MS) results found that RBM39 binds and interact with the AP-1 component c-Jun (Figures 5A, B) (14). In addition, the data displays that the cryptic autonomous transactivation domain (AD) of RBM39 combined and interacted with the basic region-leucine zipper (bZIP) of c-Jun (14). In previous studies, the interaction region of RBM39 included the end of the RRM2 domain, the whole RRM3 domain, and the sequence connecting the two domains in the middle. Therefore, we are curious about whether RBM39 was 

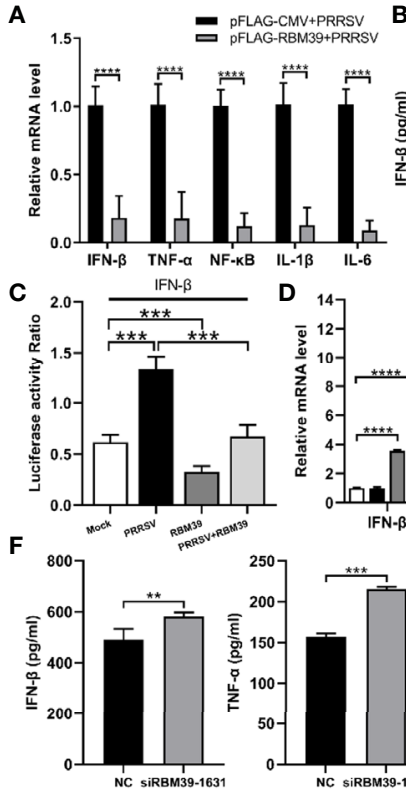
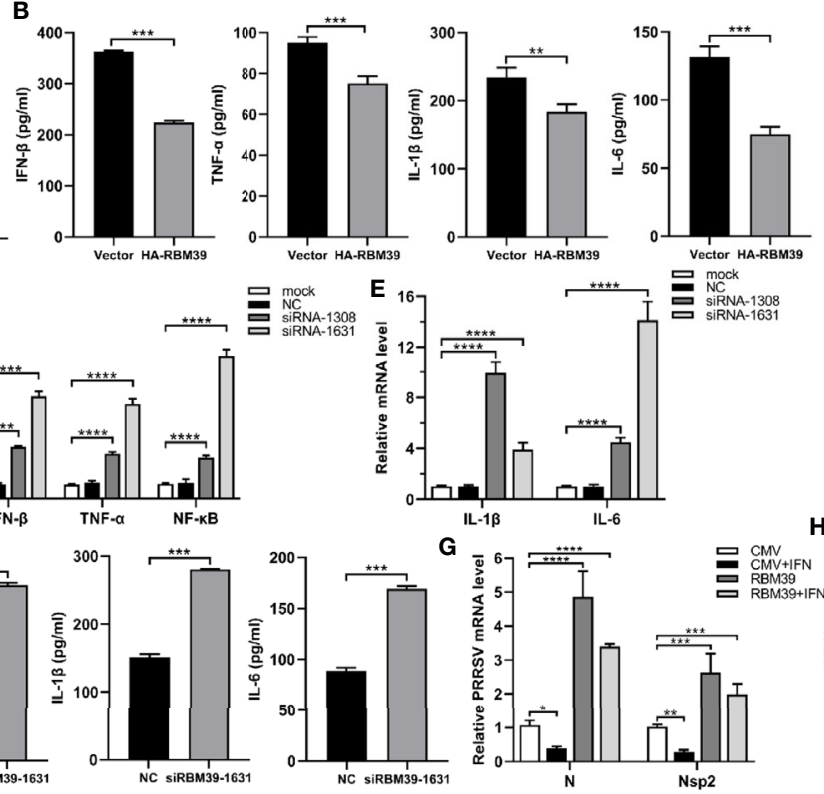

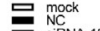
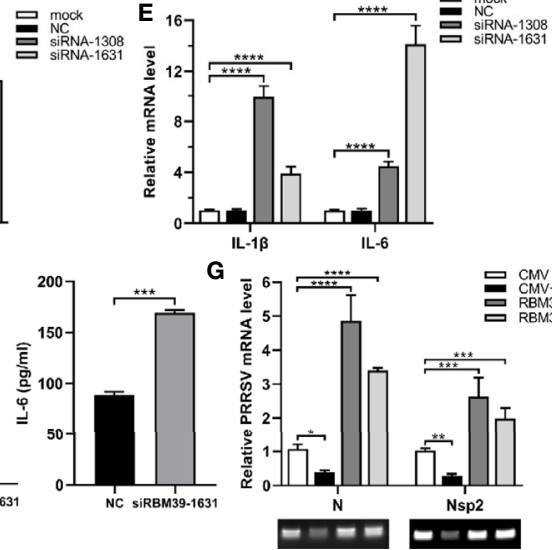

$\mathbf{H}$
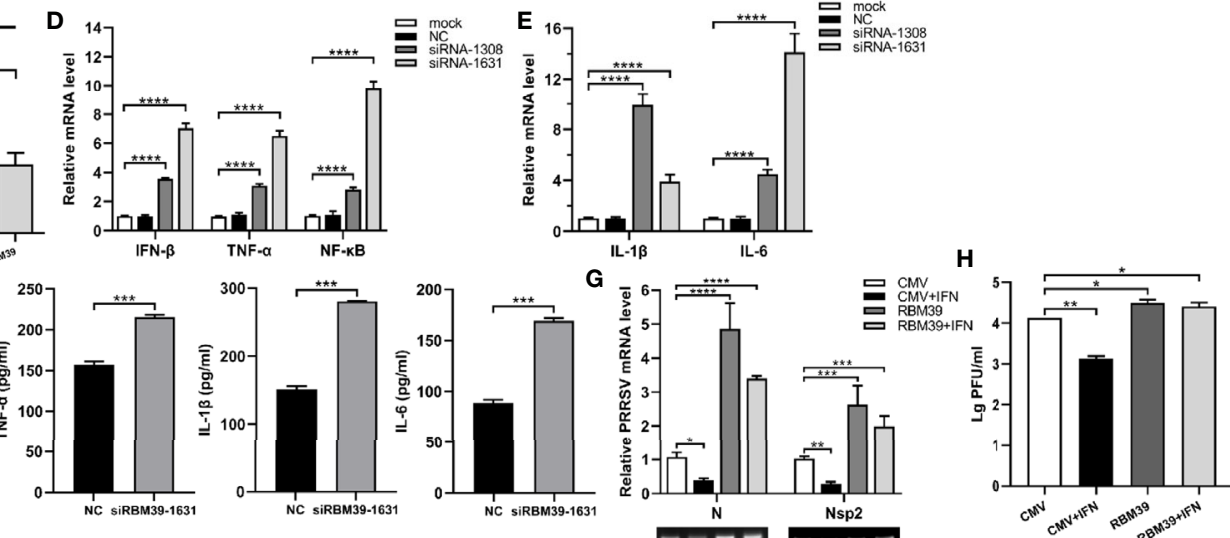

FIGURE 4 | The promotion of PRRSV proliferation by RBM39 is related to innate immunity. 3D4/21 cells were transfected with Flag-RBM39 or empty vector plasmid (A, B, G, H), siRNA of RBM39 or NC (D-F) and the infected with PRRSV $12 \mathrm{~h}$ post-transfection. The mRNA levels of IFN- $\beta$, TNF- $\alpha, N F-\kappa B$, IL-1 $\beta$, IL-6 were determined by qRT-PCR at $24 \mathrm{~h}$ post-infection (A, D, E). Cytokine detection in the condition of RBM39 overexpressed (B) or interfered (F) by ELISA kits (Solarbio). (C) HEK293 T cells were transfected or co-transfected with HA-RBM39 and PRRSV infectious clone plasmid. The luciferase activity of IFN- $\beta$ promoter reporter gene was measured through Dual Luciferase Reporter Gene Assay Kit (Yeasen Technology). (G) The cells were treated with IFN post-infection. After another $24 \mathrm{~h}$, mRNA levels of PRRSV N and nsp2 were tested by qRT-PCR. (H) Detection of viral titers of PRRSV in cell supernatants after IFN treated. Data are representative of results from three independent experiments. The statistically significant at P-value was demonstrated in Statistical Analysis.

capable of interacting with c-Jun when there is only RRM2 or RRM3. Afterwards, the plasmids HA-c-Jun and Flag-RBM39 (full-length), RRM2, RRM3, $\triangle \mathrm{RRM1}, \triangle \mathrm{RRM}$ or empty vector was co-transfected into HEK293T respectively. Subsequently, cells were harvested after $24 \mathrm{~h}$ and interaction between RBM39 and c-Jun was detected via co-immunoprecipitation. The results showed that c-Jun can interact with full-length RBM39, RRM2, RRM 3 and $\triangle R R M 1$ but not $\triangle R R M$ and empty vector (Figure 5C). It is worth noting when both RRM2 and RRM3 domains exist, the interaction between RBM39 and c-Jun is stronger than only one of them exists (Figure 5B). To observe the co-localization of the two proteins in the cell, we co-transfected plasmids Flag-RBM39+ HA-c-Jun or Flag-c-Jun + HA-RBM39 into HEK293T cell lines. Confocal microscopy showed that both RBM39 and c-Jun expressed and co-localized in the nucleus, while similar results were not observed in the control groups (Figures 5D-G).

\section{RBM39 Down-Regulates AP-1 Signaling Pathway}

The c-Jun is an important part of AP-1 signaling pathway and a significant immune-related protein. To investigate whether RBM39 impacts AP-1 signaling pathway, HEK293 T cells were transfected or co-transfected with HA-RBM39 and AP-1 promoter reporter plasmid. The results display that the activity of AP-1 promoter reporter gene was reduced by RBM39 overexpression in a dose-dependent manner (Figures 6A, B).
To investigate whether PRRSV infection can cause the intracellular localization changes of RBM39 and c-Jun in 3D4/21 cells, the cells were respectively transfected with HA-RBM39 and Flag-c-Jun plasmids, $12 \mathrm{~h}$ after transfection, cells were infected with 0.4 MOI PRRSV. In the subsequent immunofluorescence analysis, we noticed that RBM39 or c-Jun proteins were localized in the nucleus ahead of PRRSV infection (Figures 6C-E, up). After the virus infection, we observed that both RBM39 and c-Jun proteins had nuclear-cytoplasmic trafficking and co-localization (Figures 6C-E, down). These results illustrated that PRRSV infection caused the nuclear export of RBM39 and c-Jun and this phenomenon may be related to the mechanism of RBM39 promoting PRRSV proliferation.

\section{RBM39 Impair Phosphorylation of C-Jun to Down-Regulation of AP-1 Signaling Pathway}

To test whether RBM39 down-regulates the AP-1 pathway by affecting c-Jun, we designed siRNA to knock down the expression of c-Jun (Figures 7A, B). First, PRRSV infection triggers the activation of the AP-1 signaling pathway (Figures 7C, D, F), and RBM39 overexpression (Figure 7E) and c-Jun knockdown (Figures 7C, F) led to a decrease in the activity of the AP-1 promoter reporter gene (Figures 7D, E). On this basis, we hypothesized that the down-regulation of AP-1 activity is not relevant to the interaction between RBM39 with c-Jun; therefore, 


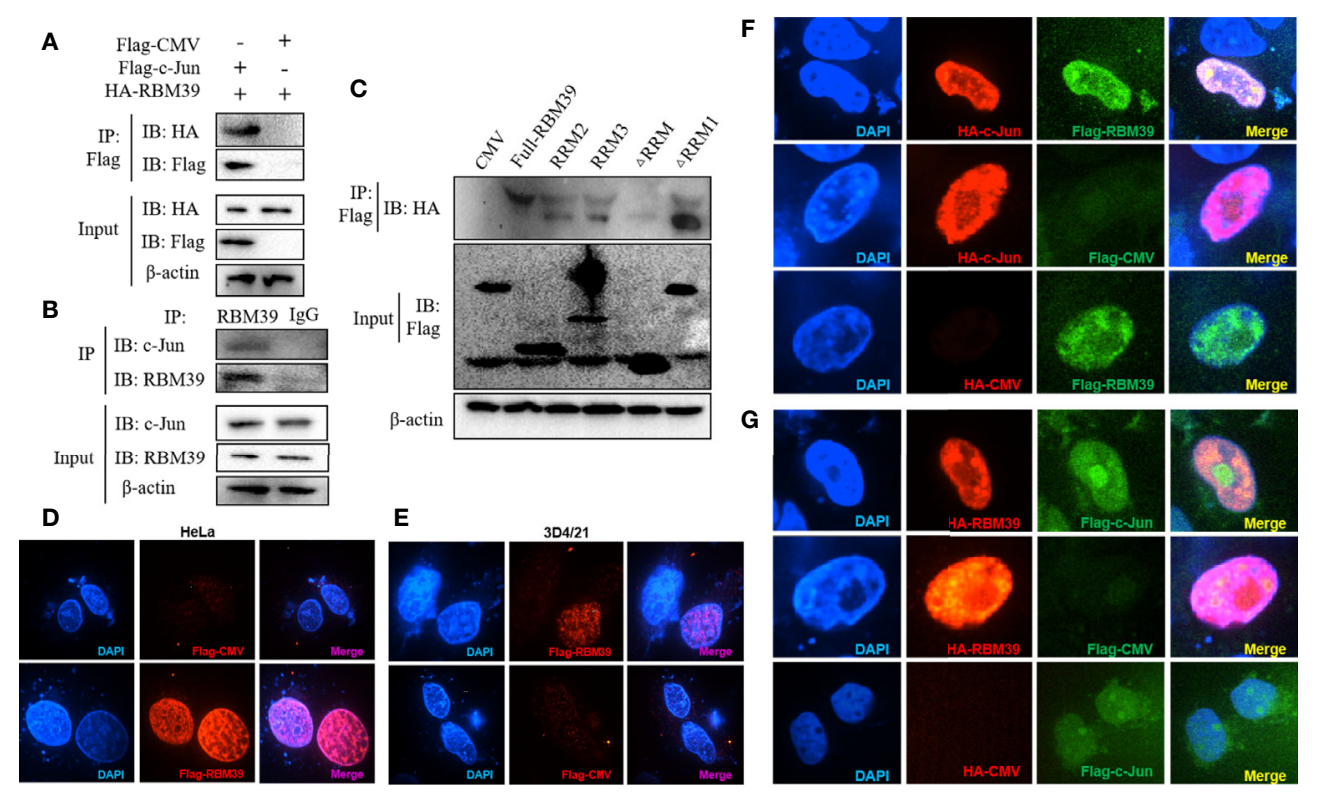

FIGURE 5 | Interaction and co-localization between RBM39 and C-Jun. Interaction between RBM39 and C-Jun in the condition of overexpressed (A) or endogenous expression (B). (C) HEK293 cells were first transfected with HA-C-Jun and then co-transfected with Flag-RBM39 (full-length), RRM2, RRM3, $\Delta$ RRM1, $\Delta$ RRM plasmid or empty vector respectively. The cell lysates were then immunoprecipitated with an anti-HA MAb and detected by Western blot at $24 \mathrm{~h}$ post-transfection. (D-G) HEK293T cell were co-transfected with plasmid Flag-RBM39+HA-c-Jun or Flag-C-Jun+HA-RBM39 plasmid. After 24 h, cells were fixed by $4 \%$ paraformaldehyde and doubly stained with rabbit anti-HA mAb and mouse anti-Flag antibody followed by FITC-conjugated goat anti-rabbit lgG (green) or IF555conjugated goat anti-mouse IgG (red) antibody. Nuclei were stained with Hoechst 33258 dye (blue). Interaction and nuclear localization of RBM39 and c-Jun were observed using Laser confocal fluorescence microscope. Data are representative of results from three independent experiments.

RBM39 and siRNA-c-Jun could reduce AP-1 activity to a greater degree than either. However, the results showed that RBM39 and siRNA-c-Jun did not have a synergistic effect with each other (Figure 7E), which indirectly indicates that the downregulation of the AP-1 pathway by RBM39 is achieved by affecting c-Jun. Similarly, the activity of AP-1 promoter reporter gene was increased by PRRSV stimuli but reduced by RBM39 and PRRSV co-transfection compared with the control group (Figures 7C, D, F). However, compared with the cotransfection of RBM39 and PRRSV, the activity of AP-1 was not significantly reduced in the additional condition of c-Jun knockdown (Figure 7F), indicating that knockdown of c-Jun has not a synergistic effect on the AP-1 activity reduction caused by RBM39 (Figure 7F), further indicating the down-regulation of the AP-1 pathway by RBM39 is related to c-Jun.

To further prove the above conclusion, we tested the effect of c-Jun knockdown and RBM39 on PRRSV proliferation. The results illustrate that it is obvious that RBM39 and siRNA-c-Jun alone can promote the proliferation of PRRSV (Figures 7G-I). However, when the RBM39 and siRNA-c-Jun were cotransfected, the promotion of PRRSV proliferation not only did not increase synergistically, but decreased, indicating that the promotion of PRRSV proliferation by RBM39 is through interacting with c-Jun (Figures 7G-I).

In order to detect the effect of RBM39 on the phosphorylation level of proteins in the cell signaling pathway caused by PRRSV infection, 3D4/21 cells were transfected or co-transfected with
PRRSV and HA-RBM39 plasmids, phosphatase Inhibitors/CIP was used to treat cell lysate supernatant and c-Jun antibody was used to detection for Western Blot analysis. In addition, phosphorylated antibody was used to detect the phosphorylation level of the protein 24 hours post-infection. The results showed that c-Jun was heavily phosphorylated after PRRSV (Figure 7J), which triggered subsequent immune responses. However, under the condition of RBM39 overexpression, the phosphorylation of c-Jun caused by PRRSV infection was greatly reduced and in a dose-dependent manner (Figures $\mathbf{7 K}, \mathbf{L}$ ). What is noteworthy is that the c-Jun and phosphorylation level of JNK did not decrease significantly in the co-transfection group (Figures $7 \mathbf{L}, \mathbf{M}$ ), indicating that RBM39 may not affect the phosphorylation of JNK and its upstream molecules triggered by PRRSV infection. In summary, the overexpression of RBM39 reduces the phosphorylation level of c-Jun after PRRSV infection, and thus down-regulates the AP-1 signaling pathway. This may be the mechanism by which RBM39 promotes PRRSV proliferation.RBM39 Binds to PRRSV RNA

RBM24, which belongs to the same RNA-binding protein family as RBM39, has RNA-binding function and can bind to the 5'- and 3'-terminal ends of HBV RNA (37). Here, the RBM39 binds to viral RNA were also identified. To verify whether RBM39 can bind to PRRSV RNA, 3D4/21 cells were transfected with HA-RBM39 or Flag-RBM39 plasmids and infected with PRRSV $12 \mathrm{~h}$ after transfection. After another 24 hours, the cells were collected and lysed with RIPA 
A

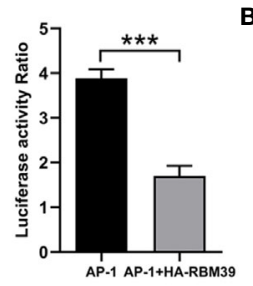

E
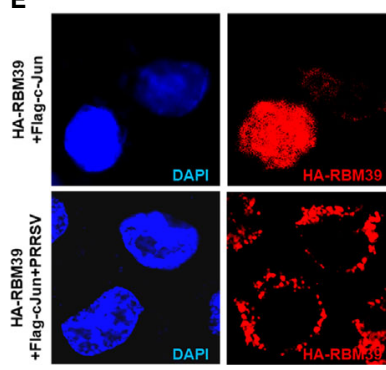

B

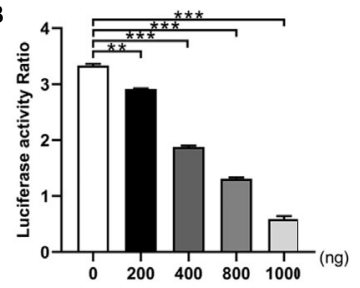

C
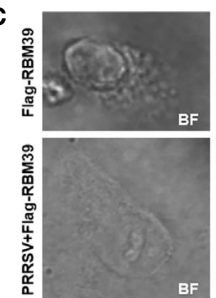

D
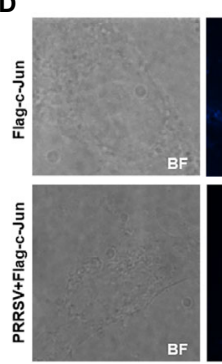
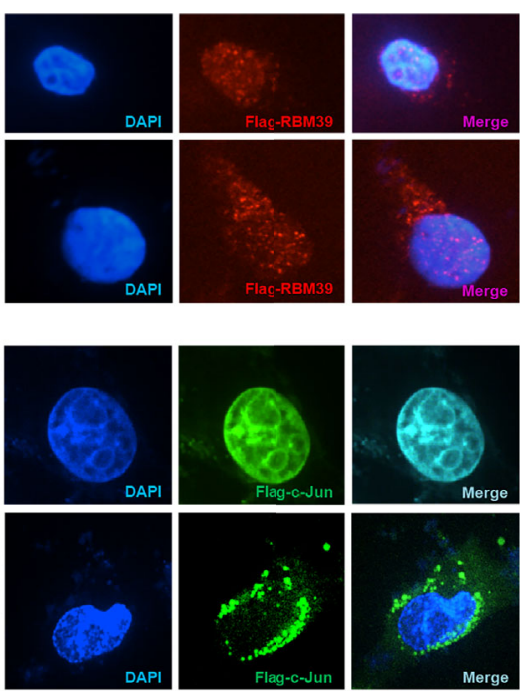

FIGURE 6 | RBM39 down-regulates AP-1 signaling pathway and PRRSV infection triggers out of the nucleus of translocation of RBM39 and C-Jun. (A, B) HEK293 T cells were transfected or co-transfected with AP-1 promoter reporter plasmid and different concentrations of HA-RBM39. The luciferase activity of AP-1 promoter reporter gene was measured through Dual Luciferase Reporter Gene Assay Kit (Yeasen Technology). (C-E) The 3D4/21cells were respectively transfected or cotransfected with HA-RBM39 and Flag-c-Jun plasmids, the cells were infected with $0.4 \mathrm{MOI}$ PRRSV $12 \mathrm{~h}$ post-transfection. After $24 \mathrm{~h}$, cells were incubated with rabbit anti-HA mAb and mouse anti-Flag antibody followed by FITC-conjugated goat anti-rabbit lgG (green) or IF555-conjugated goat anti-mouse IgG (red) antibody. Nuclei were stained with Hoechst 33258 dye (blue). Interaction and nuclear localization of RBM39 and c-Jun were observed using Laser confocal fluorescence microscope. Data are representative of results from three independent experiments. The statistically significant at P-value was demonstrated in Statistical Analysis.

supplemented with murine RNase inhibitor (40U/ $\mu$ l, Yeasen). Part of the supernatant was used for immunoblotting analysis (Figures 8A, B), and the remaining supernatant was carefully added to HA/Flag-tags beads and combined overnight at $4^{\circ} \mathrm{C}$. After RNA was extracted and reverse transcribed, it was amplified with corresponding primers, and a single band with the same size as predicted was recovered and sequenced (Figures $\mathbf{8 C}-\mathbf{E})$. Through the alignment between sequenced sequence and known sequence, we found that we correctly amplified nsp4 (85.27\%), nsp5 (87.87\%), nsp7 (67.57\%), nsp10 (81.91\%), nsp11 (91.39\%), nsp12 (74.60\%), M (92.79\%), and N (94.04\%) genes of PRRSV (Figure 8F), and indicating that RBM39 could bind to PRRSV RNA. To further explore the effects of the three RRM domains on binding to PRRSV RNA and c-Jun phosphorylation, we transfected RBM39, RRM1, RRM2, RRM3, $\Delta R R M$ plasmids, or empty vector into $3 \mathrm{D} 4 / 21$ cells. $12 \mathrm{~h}$ after transfection, cells were infected with PRRSV. After another 24 hours, the cells were collected and subjected to RIP and immunoblotting. The results show that the deletion of RRM2 or RRM3 has a significant inhibitory effect on c-Jun phosphorylation (Figure 8G), indicating that RRM2 and RRM3 may be important domains to reduce c-Jun phosphorylation. To verify whether RBM39 has a protective effect on PRRSV RNA, we heated each experimental group at $85^{\circ} \mathrm{C}$ for different time periods $(0 \mathrm{~s}, 30 \mathrm{~s}, 2 \mathrm{~min}, 5 \mathrm{~min}$, $10 \mathrm{~min}$ ) after RIP, and then level of PRRSV $\mathrm{N}$ was detected by qPCR. Presence of the full length of RBM39 or only one RRM domain, it has a certain degree of protection against PRRSV RNA. However, there is no significant difference in absence of three RRM domains (Figures 8I-M). It is worth noting that with the extension of time, the protective ability of PRRSV RNA tends to weaken overall. Moreover, we tried to amplify the RNA extracted after each group of RIP, and the results showed that nsp4, nsp5, nsp11, and N were obtained (Figure 8H), indicating that three RRM domains of RBM39 are crucial to RNA binding capacity to further impact viral proliferation.

\section{DISCUSSION}

RBM39 is an RNA-binding protein that has the function of binding RNA. In this study, we learned that RBM39 binds to PRRSV RNA and can promote its proliferation in 3D4/21 cells. According to reports, as the same RNA binding protein, RBM24 binds to the 3'-TR of HBV and stabilizes the stability of viral RNA (37). Therefore, we speculate that the upregulated cytoplasmic RBM39 promotes the combination of RBM39 with PRRSV RNA and further improve the viral RNA stability. In addition, the function of RBM39 in pre-mRNA splicing and its effects on the promotion of PRRSV proliferation should be further considered.

In summary, we report that porcine RBM39 negatively regulate the AP-1 signaling pathway and promote PRRSV proliferation. The mechanism by which RBM39 promotes PRRSV proliferation can be summarized as follows (1). After PRRSV infection, RBM39 is up-regulated, which promotes the formation of RBM39 and c-Jun complex in the cytoplasm and cytoplasmic retention. (2) PRRSV infection makes JNK phosphorylation incorporated into the nucleus, and promotes 
A

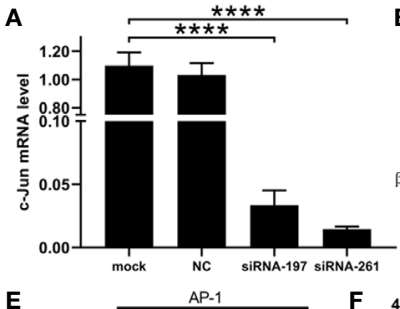

E
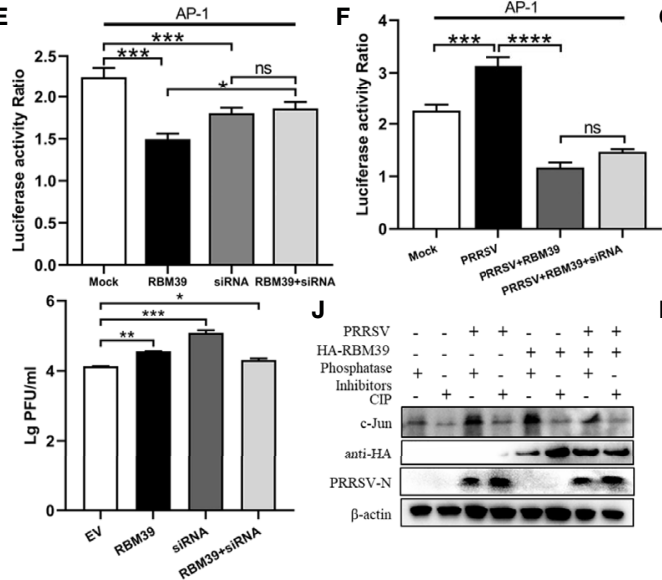

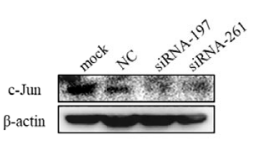

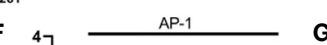

G
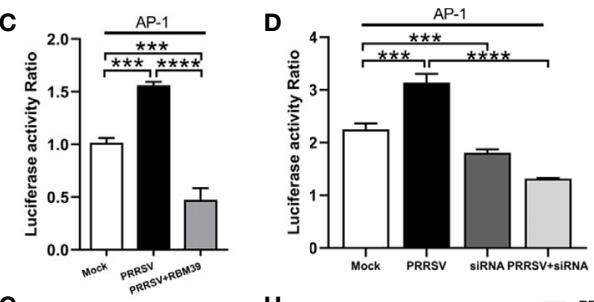

H
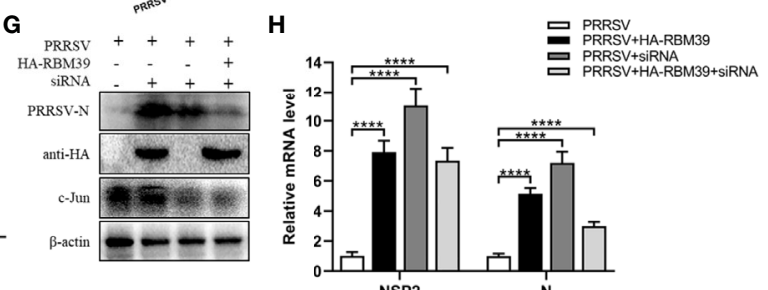

$\mathbf{K}$

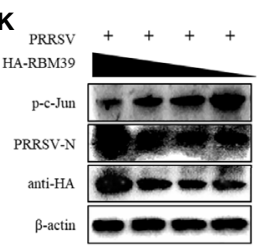

PRRST+SiRNA
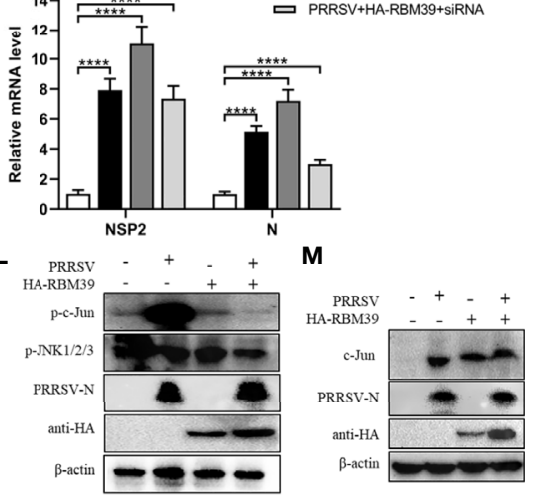

FIGURE 7 | RBM39 affect phosphorylation of C-Jun to down-regulation of AP-1 signaling pathway. 3D4/21 cells were transfected with siRNA of C-Jun or the NC, and the mRNA and protein levels of c-Jun were examined by qRT-PCR (A) and WB analysis (B). (C-F) HEK 293 T cells were transfected or co-transfected with RBM39, siRNA of C-Jun, PRRSV infectious clone plasmid, AP-1/Renilla luciferase reporter plasmids. At $24 \mathrm{~h}$ after transfection, the luciferase activity of AP-1 promoter reporter gene was measured through Dual Luciferase Reporter Gene Assay Kit (Yeasen Technology). (H, J) 3D4/21 cells were transfected or cotransfected with RBM39 and siRNA of c-Jun, respectively. The cells were infected with $0.4 \mathrm{MOI}$ PRRSV $12 \mathrm{~h}$ post-transfection and collected another 24 h postinfection. The protein levels of PRRSV N were detected by WB (G) and the mRNA levels of PRRSV N and nsp2 were tested by qRT-PCR (H). (I) Detection of viral titers of PRRSV in cell supernatants of $\mathbf{( H )}$ each experiment group samples. (J) The C-Jun protein level was detected in the condition of phosphatase Inhibitors/CIP treated by WB analysis. (K) 3D4/21 cells were transfected with different concentrations of RBM39 $(2 \mu \mathrm{g}, 1 \mu \mathrm{g}, 1.5 \mu \mathrm{g}$, $0.5 \mu \mathrm{g})$. The cell lysates were incubated with Rabbit anti-phospho-c-Jun (Ser73) mAb and detected by Western blotting. (L, M) 3D4/21 cells were transfected or untransfected with RBM39. 12 h after transfection, cells were infected or no infected with PRRSV. The protein levels of p-c-Jun, p-JNK1/2/3 (L) and c-Jun (M) were analyzed by immunoblotting. Data are representative of results from three independent experiments. The statistically significant at P-value was demonstrated in Statistical Analysis. ns, no significant difference.

the phosphorylation of nuclear transcription factor c-Jun; RBM39 and the phosphorylated c-Jun in the nucleus form a complex that is prone to translocation from the nucleus to the cytoplasm, which further reduces the retention of c-Jun in the nucleus; Decreased phosphorylated c-Jun in the nucleus, downregulating the transcription complex of c-Jun and c-Fos, resulting in down-regulation of the transcription level of IFN$\beta$, TNF- $\alpha$,IL-1 $\beta$,IL- 6 and other genes controlled by the AP-1 promoter. (3) The enhancement of the binding capacity of RBM39 with PRRSV RNA hints that the improvement of viral RNA stability also contributes to the PRRSV proliferation (Figure 9). It is worth noting that RBM39 does not markedly affect the phosphorylation level of JNK and its upstream. In addition, the activation of the RBM39 promoter is an issue worthy of discussion.

The RNA-binding protein family can promote or inhibit the proliferation of viruses, and the mechanism of action is different. For example, Pseudouridine Synthase 4 (Pus4) could compete with the capsid protein (CP) to bind to plusstrand Brome Mosaic Virus (BMV) RNAs and prevent virion formation (38). EAP (EBER-associated protein) is a cellular RNA-binding protein which recognizes conserved stem-loop structure in the Epstein-Barr virus (EBV) small RNA EBER 1 and herpesvirus papio (HVP) small RNA homologous to EBER 1 , and this may be related to regulating viral gene expression, replication and proliferation (39-42). Fátyol Károly et al. proposed the opinion that dsRNA-binding protein 2 (DRB2) of Nicotiana benthamiana plays a direct role in potato virus $\mathrm{X}$ (PVX)-elicited systemic necrosis through adaptive RNA interference (RNAi) pathway and innate pattern-triggered immune (PTI) responses, thereby participating in antiviral defense and restricting viral infections (8). Numerous doublestranded RNA binding proteins of plants and mammals, such as Arabidopsis DRB3, Drosophila R2D2, mammalian TRBP and PACT, are related to antiviral defense, and the mechanisms are different, which is worthy of further investigation $(8,11$, 43-47).

In addition, the virus itself also encodes RNA-binding protein for its pivotal role in replication, transcription, etc. The NP protein (nucleoprotein) of influenza virus is a structural singlestranded RNA binding protein, which is a key adaptor molecule between the virus and the host cell process $(48,49)$. NP not only interacts with cellular polypeptides, but also interacts with various viruses and cellular macromolecules, including RNA, 


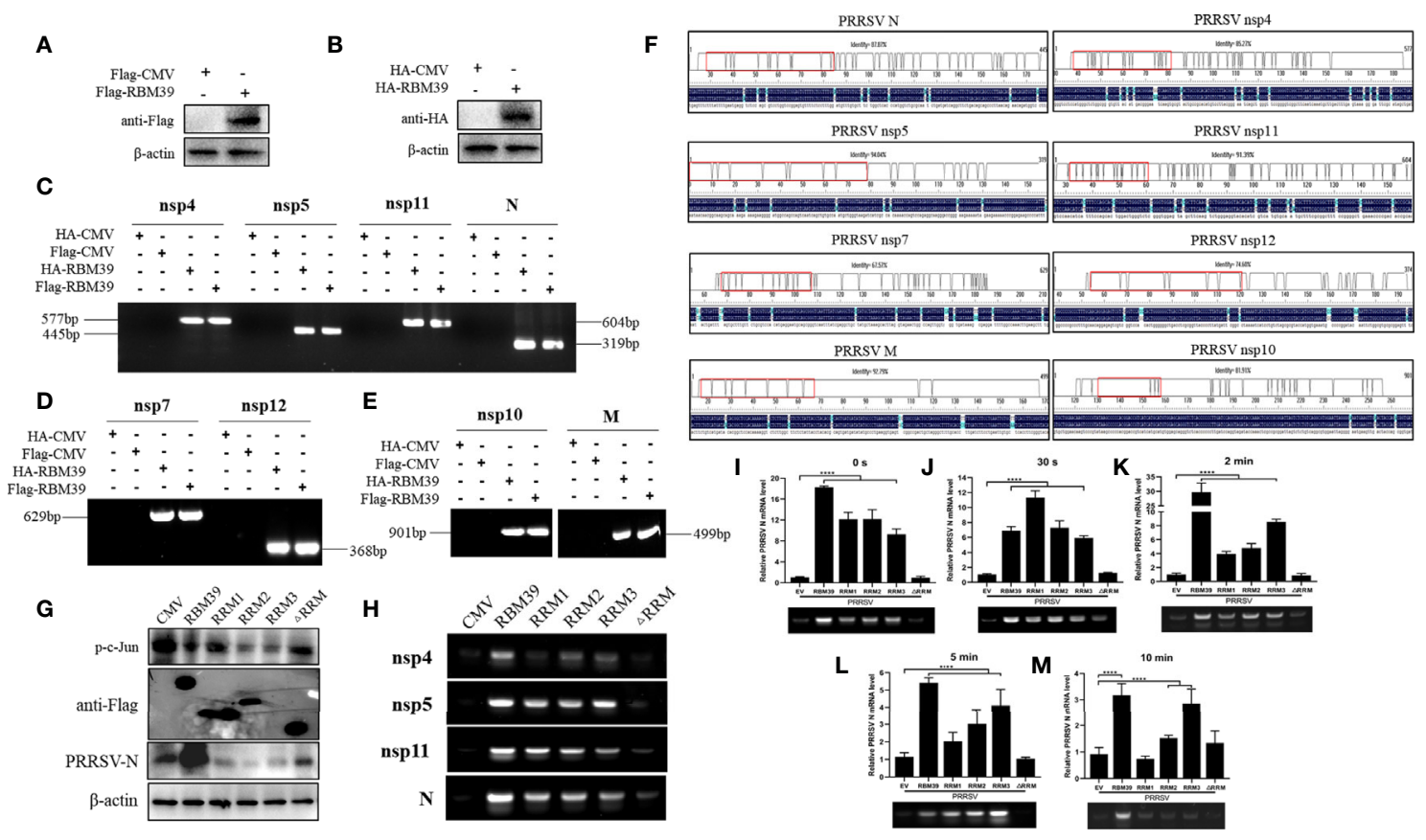

FIGURE 8 | RBM39 binds to PRRSV RNA. (A, B) 3D4/21 cells were transfected with Flag/HA-RBM39 and infected with PRRSV $12 \mathrm{~h}$ after transfection. After another 24 hours, the cells were detected for immunoblotting analysis with anti HA/Flag mAb. (C-E) The agarose gel electrophoresis of PRRSV nsp4, nsp5, nsp7, nsp10-12, M and N PCR products after extracted RNA was reverse transcribed and amplified with corresponding primers. (F) Comparison of sequencing results using DNAMAN. (G-M) 3D4/21 cells were transfected with Flag-RBM39, RRM1, RRM2, RRM3, $\triangle R R M$, or empty vector respectively and infected with PRRSV $12 \mathrm{~h}$ after transfection. After another 24 hours, the cells were tested for immunoblotting (G) and RIP analysis. (I-M) The PRRSV N level was analyzed by qRT-PCR after RIP. (H) The agarose gel electrophoresis of PRRSV nsp4, nsp5, nsp11, and N PCR products. Data are representative of results from three independent experiments. The statistically significant at P-value was demonstrated in Statistical Analysis.

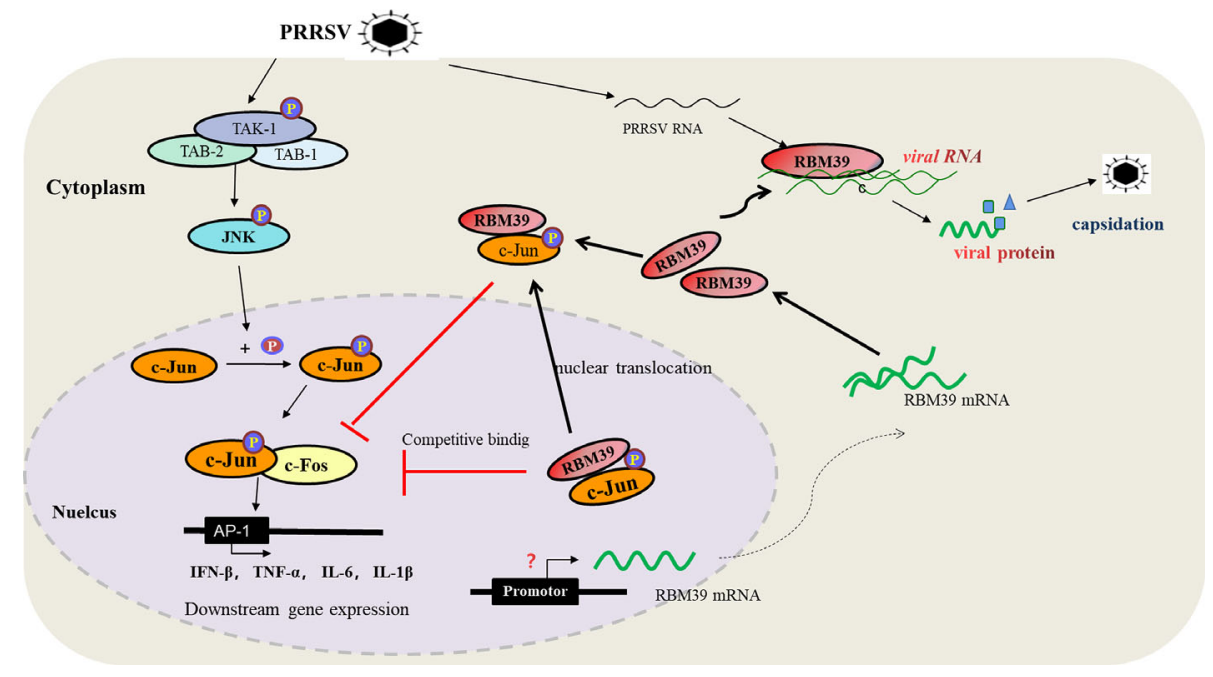

FIGURE 9 | The diagram of the mechanism of RBM39 promoting PRRSV proliferation. After PRRSV infection, the combination of RBM39 and c-Jun translocates to the outside of the nucleus together under certain effects, which hinders the phosphorylation of c-Jun by JNK and reduces the level of c-Jun phosphorylation. At the same time, RBM39 competes with c-Fos to bind c-Jun and robs c-Jun of AP-1 dimer, which reduces the number of AP-1 dimers, and then down-regulates the activity of AP-1 pathway and its downstream signals, and finally, promotes the proliferation of PRRSV. In addition, the combination of RBM39 and PRRSV RNA may promote PRRSV proliferation to some extent. 
viral matrix proteins and viral RNA-dependent RNA polymerase, which are related to the transcription, replication, and intracellular trafficking of NP in the viral genome $(48,49)$. JC, Watson et al. identified a vaccinia virus-encoded doublestranded RNA-binding protein, which may be involved in inhibition of the double-stranded RNA-dependent protein kinase induced by interferon (50) and antagonized cellular antiviral response pathways triggered by dsRNA (51).

After PRRSV infection, both RBM39 and c-Jun showed the phenomenon of translocation from the nucleus to the cytoplasm. Quantities of previous studies have shown that RNA-binding proteins have been transferred from the inside to the outside of the nucleus. Another RBP protein in the RBM family, RBM14, was relocated to the nucleolus after influenza A infection (52). CIRP is a cold-induced RNA binding protein, usually located in the nucleus; however, it will migrate into the cytoplasm under a variety of physiological or stress conditions (53). For example, when human colon cancer RKO cells are irradiated by ultraviolet light, the induced genotoxic stress causes CIRP to migrate from the nucleus to the cytoplasm (54). CIRP exerts a protective effect on cells by combining specific target genes RPA2 and TRX to enhance the stability of mRNA and the translation of specific target genes (54). When the methyltransferase I at the arginine terminal in Xenopus oocytes is overexpressed, CIRP in the nucleus will transfer to the cytoplasm and accumulate (55). Under oxidative stress conditions, vascular endothelial cells will cause the CIRP in the nucleus to undergo nuclear-cytoplasmic traversal and aggregate to the stress particles in the cytoplasm (56). When salmon is under hyperosmotic stress, it will induce the production of CIRP homologue SGRP, which has the function of nuclear-cytoplasmic trafficking, allowing it to migrate into the cytoplasm (57). As predicted by the online website http://www.cbs.dtu.dk/services/NetNES/, neither RBM39 nor c-Jun has NES (leucine-rich nuclear export signals) sequences. Therefore, after PRRSV infection, the translocation of them to the cytoplasm may involve other proteins containing the NES sequence, which leading to the translocation of the two.

In higher eukaryotes, the production and expression of mature gene mRNA is a complex process that includes initiation of transcription, 5'-capping, splicing, and polyadenylation (58). RNA processing events play a vital role for specific transcription factors in pre-mRNA processing (59). Research have shown that RBM39 can regulated pre-mRNA processing in a steroid hormone

\section{REFERENCES}

1. Pagliuso A, Tham TN, Allemand E, Robertin S, Dupuy B, Bertrand Q, et al. An RNA-Binding Protein Secreted by a Bacterial Pathogen Modulates Rig-I Signaling. Cell Host Microbe (2019) 26:823-35.e11. doi: 10.1016/ j.chom.2019.10.004

2. Del Gatto-Konczak F, Bourgeois FC, Le Guiner C, Kister L, Gesnel CM, Stévenin J, et al. The RNA-Binding Protein TIA-1 is a Novel Mammalian Splicing Regulator Acting Through Intron Sequences Adjacent to a 5' Splice Site. Mol Cell Biol (2000) 20:6287-99. doi: 10.1128/mcb.20.17.6287-6299.2000

3. Grifone R, Shao M, Saquet A, Shi DL. Rna-Binding Protein Rbm24 as a Multifaceted Post-Transcriptional Regulator of Embryonic Lineage receptor-dependent manner (58) and knockdown of RBM39 via siRNA changes the splice form of vascular endothelial growth factor A (VEGF-A) $(58,60,61)$. Therefore, the mechanism of the precursor RNA splicing function of RBM39 and other RNA binding proteins after virus infection is worth exploring.

\section{DATA AVAILABILITY STATEMENT}

The raw data supporting the conclusions of this article will be made available by the authors, without undue reservation.

\section{AUTHOR CONTRIBUTIONS}

Conceived and designed the experiments: JH. Performed the experiments: YS, XL, YG, MZ, RS, JS, ZT, and LZ. Analyzed the data: YS and JH. Contributed reagents/materials: JH. Wrote the paper: YS and JH. All authors contributed to the article and approved the submitted version.

\section{FUNDING}

This work was supported by the National Key Research and Development Program of China (2018YFD0500500) and the key underprop project of Tianjin Science and Technology Bureau in China (20YFZCSN00340).

\section{ACKNOWLEDGMENTS}

We would like to thank the key underprop project of Tianjin Science and Technology Bureau in China (20YFZCSN00340) and the National Key Research and Development Program of China (2018YFD0500500).

\section{SUPPLEMENTARY MATERIAL}

The Supplementary Material for this article can be found online at: https://www.frontiersin.org/articles/10.3389/fimmu.2021.664417/ full\#supplementary-material

Differentiation and Cellular Homeostasis. Cells (2020) 9(8):1891. doi: 10.3390/cells9081891

4. Christou-Kent M, Dhellemmes M, Lambert E, Ray PF, Arnoult C. Diversity of RNA-Binding Proteins Modulating Post-Transcriptional Regulation of Protein Expression in the Maturing Mammalian Oocyte. Cells (2020) 9:9030662. doi: 10.3390/cells9030662

5. Lewei Z, Qifa YE. Research Advance on Effects of RNA Binding Motif3 (RBM3). Med J Wuhan Univ (2019) 05:850-5. doi: CNKI:SUN:SLKZ.0.201406-010

6. Carey BD, Akhrymuk I, Dahal B, Pinkham CL, Bracci N, Finstuen-Magro S, et al. Protein Kinase C Subtype Delta Interacts With Venezuelan Equine Encephalitis Virus Capsid Protein and Regulates Viral RNA Binding Through 
Modulation of Capsid Phosphorylation. PloS Pathog (2020) 16:e1008282. doi: 10.1371/journal.ppat.1008282

7. Hojo H, Yashiro Y, Noda Y, Ogami K, Yamagishi R, Okada S, et al. The RNAbinding Protein QKI-7 Recruits the Poly(a) Polymerase GLD-2 for 3' Adenylation and Selective Stabilization of Microrna-122. J Biol Chem (2020) 295:390-402. doi: 10.1074/jbc.RA119.011617

8. Fátyol K, Fekete KA, Ludman M. Double-Stranded-Rna-Binding Protein 2 Participates in Antiviral Defense. J Virol (2020) 94:e00017-20. doi: 10.1128/ JVI.00017-20

9. Yin S, Huo Y, Dong Y, Fan L, Yang H, Wang L, et al. Activation of C-Jun NH (2)-terminal Kinase is Required for Porcine Reproductive and Respiratory Syndrome Virus-Induced Apoptosis But Not for Virus Replication. Virus Res (2012) 166:103-8. doi: 10.1016/j.virusres.2012.03.010

10. Laver JD, Ly J, Winn AK, Karaiskakis A, Lin S, Nie K, et al. The RNA-Binding Protein Rasputin/G3BP Enhances the Stability and Translation of Its Target Mrnas. Cell Rep (2020) 30:3353-67.e7. doi: 10.1016/j.celrep.2020.02.066

11. Hentze MW, Castello A, Schwarzl T, Preiss T. A Brave New World of RNAbinding Proteins. Nat Rev Mol Cell Biol (2018) 19:327-41. doi: 10.1038/ nrm. 2017.130

12. Metrick CM, Koenigsberg AL, Heldwein EE. Conserved Outer Tegument Component UL11 From Herpes Simplex Virus 1 Is an Intrinsically Disordered, RNA-Binding Protein. mBio (2020) 11:e00810-20. doi: 10.1128/ mBio.00810-20

13. Dutta J, Fan G, Gelinas C. Caper $\alpha$ Is a Novel Rel-Tad-Interacting Factor That

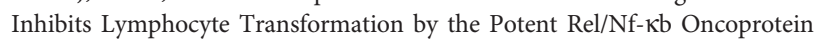
V-Rel. J Virol (2008) 82:10792-802. doi: 10.1128/JVI.00903-08

14. Jung DJ, Na SY, Na DS, Lee JW. Molecular Cloning and Characterization of CAPER, a Novel Coactivator of Activating Protein-1 and Estrogen Receptors. J Biol Chem (2002) 277:1229-34. doi: 10.1074/jbc.M110417200

15. Han T, Goralski M, Gaskill N, Capota E, Kim J, Ting TC, et al. Anticancer Sulfonamides Target Splicing by Inducing RBM39 Degradation Via Recruitment to DCAF15. Science (2017) 356(6336):eaal3755. doi: 10.1126/ science.aal 3755

16. Stepanyuk GA, Serrano P, Peralta E, Farr CL, Axelrod HL, Geralt M, et al. Uhm-ULM Interactions in the RBM39-U2AF65 Splicing-Factor Complex. Acta Crystallogr D Struct Biol (2016) 72:497-511. doi: 10.1107/ S2059798316001248

17. Chilewski SD, Bhosale D, Dees S, Hutchinson I, Jasmin JF. Development of CAPER Peptides for the Treatment of Triple Negative Breast Cancer. Cell Cycle (Georgetown Tex) (2020) 19:1-16. doi: 10.1080/15384101. 2020.1711579

18. Faherty N, Benson M, Sharma E, Lee A, Howarth A, Lockstone H, et al. Negative Autoregulation of BMP Dependent Transcription by SIN3B Splicing Reveals a Role for RBM39. Sci Rep (2016) 6:28210. doi: 10.1038/srep28210

19. Karin M, Liu ZG, Zandi E. AP-1 Function and Regulation. Curr Opin Cell Biol (1997) 9:240-6. doi: 10.1016/S0955-0674(97)80068-3

20. Corteggio A, Urraro C, Roperto S, Roperto F, Borzacchiello G. Phosphatidylinositol-3-Kinase-Akt Pathway, Phospho-JUN and PhosphoJNK Expression in Spontaneously Arising Bovine Urinary Bladder Tumours. J Comp Pathol (2010) 143:173-8. doi: 10.1016/j.jcpa.2010.03.001

21. Angel P, Karin M. The Role of Jun, Fos and the AP-1 Complex in CellProliferation and Transformation. Biochim Et Biophys Acta Rev Cancer (1991) 1072(2-3):129-57. doi: 10.1016/0304-419x(91)90011-9

22. Jochum W, Passegué E, Wagner EF. AP-1 in Mouse Development and Tumorigenesis. Oncogene (2001) 20:2401-12. doi: 10.1038/sj.onc.1204389

23. Hai TW, Liu F, Allegretto EA, Karin M, Green MR. A Family of Immunologically Related Transcription Factors That Includes Multiple Forms of ATF and AP-1. Genes Dev (1988) 2:1216. doi: 10.1101/gad.2.10.1216

24. Carrillo RJ, Dragan AI, Privalov PL. Stability and DNA-binding Ability of the bZIP Dimers Formed by the ATF-2 and c-Jun Transcription Factors. J Mol Biol (2010) 396:431-40. doi: 10.1016/j.jmb.2009.11.050

25. Pulverer BJ, Kyriakis JM, Avruch J, Nikolakaki E, Woodgett JR. Phosphorylation of c-Jun Mediated MAP Kinases. Nature (1991) 353:6704. doi: $10.1038 / 353670 \mathrm{a} 0$

26. Su Y, Shi P, Zhang L, Lu D, Zhao C, Li R, et al. The Superimposed Deubiquitination Effect of OTULIN and Porcine Reproductive and Respiratory Syndrome Virus (Prrsv) Nsp11 Promotes Multiplication of PRRSV. J Virol (2018) 92:e00175-18. doi: 10.1128/JVI.00175-18
27. Wei ZY, Wang XB, Zhang HY, Yang CH, Wang YB, Xu DH, et al. Inhibitory Effects of Indigowoad Root Polysaccharides on Porcine Reproductive and Respiratory Syndrome Virus Replication In Vitro. Antiviral Ther (2011) 16:357-63. doi: 10.3851/IMP1755

28. Xiao X, Zhang W, Hua D, Zhang L, Zhang L. Cold-Inducible RNA-binding Protein (CIRBP) Promotes Porcine Reproductive and Respiratory Syndrome Virus (PRRSV)-Induced Inflammatory Response. Int Immunopharmacol (2020) 86:106728. doi: 10.1016/j.intimp.2020.106728

29. Guo Y, Li R, Tan Z, Shi J, Fu Y, Song Y, et al. E3 Ubiquitin Ligase ASB8 Negatively Regulates Interferon Via Regulating TBK1/IKKi Homeostasis. Mol Immunol (2020) 121:195-203. doi: 10.1016/j.molimm.2020.03.011

30. Shi P, Guo Y, Su Y, Zhu M, Fu Y, Chi H, et al. Sumoylation of DDX39A Alters Binding and Export of Antiviral Transcripts to Control Innate Immunity. J Immunol (2020) 205:168-80. doi: 10.4049/jimmunol.2000053

31. Halbur PG, Evans R, Hagemoser WA, Pallares FJ, Rathje JA, Paul PS, et al. Effects of Different Us Isolates of Porcine Reproductive and Respiratory Syndrome Virus (PRRSV) on Blood and Bone Marrow Parameters of Experimentally Infected Pigs. Vet Rec (2002) 151:344-8. doi: 10.1136/ vr.151.12.344

32. Dokland T. The Structural Biology of PRRSV. Virus Res (2010) 154:86-97. doi: 10.1016/j.virusres.2010.07.029

33. Conzelmann KK, Visser N, Woensel PV, Thiel H-J. Molecular Characterization of Porcine Reproductive and Respiratory Syndrome Virus, a Member of the Arterivirus Group. Virology (1993) 193:329. doi: 10.1006/ viro.1993.1129

34. Snijder EJ, Meulenberg JJ. The Molecular Biology of Arteriviruses. J Gen Virol (1998) 79( Pt 5):961-79. doi: 10.1099/0022-1317-79-5-961

35. Ziebuhr J, Snijder EJ, Gorbalenya AE. Virus-encoded Proteinases and Proteolytic Processing in the Nidovirales. J Gen Virol (2000) 81:853-79. doi: 10.1099/0022-1317-81-4-853

36. Den BJ, Faaberg K, Meulenberg J, Wassenaar A, Plagemann P, Gorbalenya A, et al. Processing and Evolution of the N-terminal Region of the Arterivirus Replicase ORF1a Protein: Identification of Two Papainlike Cysteine Proteases. J Virol (1995) 69:4500-5. doi: 10.1128/JVI.69.7.4500-4505.1995

37. Cao H, Zhao K, Yao Y, Guo J, Gao X, Yang Q, et al. RNA Binding Protein 24 Regulates the Translation and Replication of Hepatitis C Virus. Protn Cell (2018) 9:930-44. doi: 10.1007/s13238-018-0507-x

38. Zhu J, Gopinath K, Murali A, Yi G, Hayward SD, Zhu H, et al. RNA-Binding Proteins That Inhibit RNA Virus Infection. Proc Natl Acad ences USA (2007) 104:3129-34. doi: 10.1073/pnas.0611617104

39. Toczyski DP, Steitz JA. EAP, a Highly Conserved Cellular Protein Associated With Epstein-Barr Virus Small RNAs (Ebers). EMBO J (1991) 10:459-66. doi: 10.1002/j.1460-2075.1991.tb07968.x

40. Nucifora G, Begy C, Erickson R, Drabkin HA, Rowley JD. The 3;21 Translocation in Myelodysplasia Results in a Fusion Transcript Between the AML1 Gene and the Gene for EAP, a Highly Conserved Protein Associated With the Epstein-Barr Virus Small RNA Eber 1. Proc Natl Acad Sci (1993) 90:7784-8. doi: 10.1073/pnas.90.16.7784

41. Toczyski DP, Steitz JA. The Cellular RNA-binding Protein EAP Recognizes a Conserved Stem-Loop in the Epstein-Barr Virus Small RNA Eber 1. Mol Cell Biol (1993) 13:703-10. doi: 10.1128/mcb.13.1.703

42. Lee N, Yario TA, Gao JS, Steitz JA. EBV Noncoding RNA EBER2 Interacts With Host RNA-binding Proteins to Regulate Viral Gene Expression. Proc Natl Acad Sci (2016) 113:3221-6. doi: 10.1073/pnas.1601773113

43. Raja P, Jackel JN, Li S, Heard IM, Bisaro DM. Arabidopsis Double-Stranded Rna Binding Protein DRB3 Participates in Methylation-Mediated Defense Against Geminiviruses. J Virol (2014) 88:2611-22. doi: 10.1128/JVI.02305-13

44. Saunders LR, Barber GN. The dsRNA Binding Protein Family: Critical Roles, Diverse Cellular Functions. FASEB J (2003) 17:961-83. doi: 10.1096/fj.020958rev

45. Q Liu , Rand TA, Kalidas S, Du F, Kim H-E, Smith DP, et al. R2D2, a Bridge Between the Initiation and Effector Stepsof the Drosophila Rnai Pathway. Science (2003) 301:1921-5. doi: 10.1126/science.1088710

46. Benkirane M. Oncogenic Potential of TAR RNA Binding Protein TRBP and its Regulatory Interaction With RNA-dependent Protein Kinase PKR. EMBO $J$ (2014) 16:611-24. doi: 10.1093/emboj/16.3.611

47. Chi-Ping C, Chun-Kit Y, Hinson PH, Sin-Yee F, Pak-Yin L, Honglin C, et al. Antiviral Activity of Double-Stranded RNA-binding Protein PACT Against 
Influenza A Virus Mediated Via Suppression of Viral RNA Polymerase. FASEB J (2018) 32:fj.201701361R. doi: 10.1096/fj.201701361R

48. Portela AN, Digard P. The Influenza Virus Nucleoprotein: A Multifunctional RNA-binding Protein Pivotal to Virus Replication. J Gen Virol (2002) 83:72334. doi: 10.1099/0022-1317-83-4-723

49. Galarza J, Sowa A, Hill VM, Skorko R, Summers DF. Influenza A Virus NP Protein Expressed in Insect Cells by a Recombinant Baculovirus is Associated With a Protein Kinase Activity and Possesses Single-Stranded RNA Binding Activity. Virus Res (1992) 24:91-106. doi: 10.1016/0168-1702(92)90033-6

50. Watson JC, Chang HW, Jacobs BL. Characterization of a Vaccinia VirusEncoded Double-Stranded RNA-binding Protein That may be Involved in Inhibition of the Double-Stranded RNA-dependent Protein Kinase. Virology (1991) 185:206-16. doi: 10.1016/0042-6822(91)90768-7

51. KIONG HO C, Shuman S. Physical and Functional Characterization of the Double-Stranded RNA Binding Protein Encoded by the Vaccinia Virus E3 Gene. Virology (1996) 217:272-84. doi: 10.1006/viro.1996.0114

52. Beyleveld G, Chin DJ, Del Olmo EM, Carter J, Najera I, Cillóniz C, et al. Nucleolar Relocalization of RBM14 by Influenza A Virus NS1 Protein. mSphere (2018) 3(6):e00549-18. doi: 10.1128/mSphereDirect.00549-18

53. Liu Y, Hu W, Murakawa Y, Yin J, Gang W, Landthaler M, et al. ColdInduced RNA-binding Proteins Regulate Circadian Gene Expression by Controlling Alternative Polyadenylation. Sci Rep (2013) 3:2054. doi: 10.1038/srep02054

54. Yang C, Carrier F. The UV-inducible RNA-Binding Protein A18 (A18 Hnrnp) Plays a Protective Role in the Genotoxic Stress Response. J Biol Chem (2001) 276:47277-84. doi: 10.1074/jbc.M105396200

55. Aoki K, Ishii Y, Matsumoto K, Tsujimoto M. Methylation of Xenopus CIRP2 Regulates its Arginine- and Glycine-Rich Region-Mediated Nucleocytoplasmic Distribution. Nucleic Acids Res (2003) 30:5182-92. doi: 10.1093/nar/gkf638

56. Ciuzan O, Hancock J, Pamfil D, Wilson I, Ladomery M. The Evolutionarily Conserved Multifunctional Glycine-Rich RNA-binding Proteins Play Key
Roles in Development and Stress Adaptation. Physiol Plant (2014) 153:111. doi: $10.1111 /$ ppl.12286

57. Pan F, Zarate J, Choudhury A, Rupprecht R, Bradley TM. Osmotic Stress of Salmon Stimulates Upregulation of a Cold Inducible RNA Binding Protein (CIRP) Similar to That of Mammals and Amphibians. Biochimie (2004) 86:451-61. doi: 10.1016/j.biochi.2004.06.006

58. Dowhan DH, Hong EP, Auboeuf D, Dennis AP, O’Malley BW. Steroid Hormone Receptor Coactivation and Alternative RNA Splicing by U2AF65related Proteins CAPERalpha and Caperbeta. Mol Cell (2005) 17:429-39. doi: 10.1016/j.molcel.2004.12.025

59. Goldstrohm AC, Greenleaf AL, Garcia-Blanco MA. Co-transcriptional Splicing of Pre-Messenger RNAs: Considerations for the Mechanism of Alternative Splicing. Gene (2001) 277:31-47. doi: 10.1016/s0378-1119(01)00695-3

60. Uehara T, Minoshima Y, Sagane K, Sugi NH, Mitsuhashi KO, Yamamoto N, et al. Selective Degradation of Splicing Factor Caper $\alpha$ by Anticancer Sulfonamides. Nat Chem Biol (2017) 13(6):675-80. doi: 10.1038/nchembio.2363

61. Huang G, Zhou Z, Wang H, Kleinerman ES. Caper- $\alpha$ Alternative Splicing Regulates the Expression of Vascular Endothelial Growth Factor in Ewing Sarcoma Cells. Cancer (2012) 118:2106-16. doi: 10.1002/cncr.26488

Conflict of Interest: The authors declare that the research was conducted in the absence of any commercial or financial relationships that could be construed as a potential conflict of interest.

Copyright (c) 2021 Song, Guo, Li, Sun, Zhu, Shi, Tan, Zhang and Huang. This is an open-access article distributed under the terms of the Creative Commons Attribution License (CC BY). The use, distribution or reproduction in other forums is permitted, provided the original author(s) and the copyright owner(s) are credited and that the original publication in this journal is cited, in accordance with accepted academic practice. No use, distribution or reproduction is permitted which does not comply with these terms. 